\title{
On linear independence measures of the values of Mahler functions
}

\author{
Keijo Väänänen Wen Wu
}

May 22, 2018

\begin{abstract}
In this paper, we estimate the linear independence measures for the values of a class Mahler functions of degree one and two. For the purpose, we study the determinants of suitable Hermite-Padé approximation polynomials. Based on the non-vanishing of these determinants, we apply the functional equations to get an infinite sequence of approximations which is used to produce the linear independence measures.
\end{abstract}

\section{Introduction and results}

In the present work our aim is to obtain linear independence measures for the values of a class of Mahler functions $F(z), G(z) \in \mathbb{Q}[[z]]$ converging on some open disc $D_{r}:=\{z:|z|<r \leq 1\}$ and satisfying a system of Mahler type functional equations

$$
\left\{\begin{array}{l}
F\left(z^{d}\right)=p_{11}(z) F(z)+p_{12}(z) G(z)+p_{10}(z), \\
G\left(z^{d}\right)=p_{21}(z) F(z)+p_{22}(z) G(z)+p_{20}(z)
\end{array}\right.
$$

with $p_{i j}(z) \in \mathbb{Q}(z)$ satisfying $p_{11}(z) p_{22}(z)-p_{12}(z) p_{21}(z) \neq 0$. Note that Mahler functions of degree one or two satisfy functional equations of the above type, if $F(z)$ and $G(z)$ are Mahler functions of degree one, then $p_{12}(z)=p_{21}(z)=0$, and if $F(z)$ is of degree two, then we choose $G(z)=F\left(z^{d}\right)$. Our general result (Theorem 6 in Sec. 4) needs some technical notations to be presented later, and therefore to introduce our results we give here applications to some well-known functions.

The linear independence measures studied here are lower bounds for linear forms (in 1 and certain numbers $\gamma_{1}$ and $\gamma_{2}$ ) of the form

$$
\left|h_{0}+h_{1} \gamma_{1}+h_{2} \gamma_{2}\right|>C H^{-\mu}
$$

valid for any integers $h_{0}, h_{1}, h_{2}$, not all zero, where the exponent $\mu$ is given explicitly, $H=$ $\max \left\{\left|h_{1}\right|,\left|h_{2}\right|, H_{0}\right\}$, and positive constants $C$ and $H_{0}$ are independent of $h_{i}$. In our results $\gamma_{1}$ and $\gamma_{2}$ are the values of the functions under consideration at rational points $a / b \in D_{r} \backslash\{0\}$, where $\log |a|=\lambda \log b(0 \leq \lambda<\log (r b) / \log b)$. We note that generally [12, Theorem 4.4.1] implies the existence of a $\mu(\geq 2)$ in our cases below, and here our aim is to obtain an explicit upper bound for the linear independence exponent

$$
\mu\left(\gamma_{1}, \gamma_{2}\right):=\inf \left\{\mu:(2) \text { holds for some } C>0, H_{0}>0\right\} .
$$

This work is a continuation to [14, where we studied simultaneous approximations of similar numbers $\gamma_{1}$ and $\gamma_{2}$. We also note that, after Bugeaud's remarkable work [4] on Thue-Morse numbers, there has appeared several works on the irrationality exponents of the values of degree one Mahler functions, see [2, 5, 9, 11, 13, 15] and the references in [5]. In particular, the irrationality exponents of the numbers in Theorem 1-3 below equal 2 . 


\subsection{Thue-Morse number and its square}

Our first result studies the product

$$
T(z)=\prod_{j=0}^{\infty}\left(1-z^{2^{j}}\right)
$$

the generating function of the Thue-Morse sequence on $\{-1,1\}$, satisfying

$$
T(z)=(1-z) T\left(z^{2}\right) .
$$

Theorem 1. We have

$$
\mu\left(T\left(\frac{1}{b}\right), T^{2}\left(\frac{1}{b}\right)\right) \leq \frac{91}{32} \approx 2.843 \ldots
$$

More generally, if $0 \leq \lambda<7 / 29$, then

$$
\mu\left(T\left(\frac{a}{b}\right), T^{2}\left(\frac{a}{b}\right)\right) \leq \frac{91}{32-104 \lambda} .
$$

\subsection{Stern's sequence and its twisted version}

Next, let $A(z)$ and $B(z)$ be generating functions of Stern's diatomic sequence and its twisted version. These functions satisfy functional equations

$$
A(z)=\left(1+z+z^{2}\right) A\left(z^{2}\right), \quad B(z)=2-\left(1+z+z^{2}\right) B\left(z^{2}\right),
$$

of type (I), see e.g. [7].

Theorem 2. We have

$$
\mu\left(A\left(\frac{1}{b}\right), B\left(\frac{1}{b}\right)\right) \leq \frac{26}{9} \approx 2.888 \ldots
$$

More generally,

$$
\mu\left(A\left(\frac{a}{b}\right), B\left(\frac{a}{b}\right)\right) \leq \begin{cases}\frac{130}{45-149 \lambda}, & \text { if } \lambda<\frac{145}{1289}, \\ \frac{69}{25-89 \lambda}, & \text { if } \frac{145}{1289} \leq \lambda<\frac{5}{29} .\end{cases}
$$

\subsection{Lambert series $G_{3}(z)$ and $F_{3}(z)$}

The functions

$$
G_{3}(z)=\sum_{j=0}^{\infty} \frac{z^{3^{j}}}{1-z^{3^{j}}}, \quad F_{3}(z)=\sum_{j=0}^{\infty} \frac{z^{3^{j}}}{1+z^{3^{j}}}=-G_{3}(-z)
$$

satisfy

$$
(1-z) G_{3}(z)-(1-z) G_{3}\left(z^{3}\right)-z=0, \quad(1+z) F_{3}(z)-(1+z) F_{3}\left(z^{3}\right)-z=0 .
$$

The following result studies the values of these typical examples of Mahler functions. 
Theorem 3. We have

$$
\mu\left(G_{3}\left(\frac{1}{b}\right), F_{3}\left(\frac{1}{b}\right)\right) \leq \frac{129}{37} \approx 3.486 \ldots
$$

More generally,

$$
\mu\left(G_{3}\left(\frac{a}{b}\right), F_{3}\left(\frac{a}{b}\right)\right) \leq \begin{cases}\frac{129}{37-119 \lambda}, & \text { if } \lambda<\frac{25}{443}, \\ \frac{83}{24-80 \lambda}, & \text { if } \frac{25}{443} \leq \lambda<\frac{43}{337} \\ \frac{57}{17-59 \lambda}, & \text { if } \frac{43}{337} \leq \lambda<\frac{7}{29}\end{cases}
$$

\subsection{The Rudin-Shapiro sequence}

Let $\left(r_{n}\right)_{n \geq 0}$ be the Rudin-Shapiro sequence defined by $r_{0}=1, r_{2 n}=r_{n}, r_{2 n+1}=(-1)^{n} r_{n}$. Its generating function $R(z)=\sum_{n \geq 0} r_{n} z^{n}$ satisfies

$$
R(z)=R\left(z^{2}\right)+z R\left(-z^{2}\right) .
$$

We shall investigate the values of $R(z)$ and $R(-z)$ at some rational points.

Theorem 4. We have

$$
\mu\left(R\left(\frac{1}{b}\right), R\left(-\frac{1}{b}\right)\right) \leq \frac{13}{4}=3.25
$$

More generally,

$$
\mu\left(R\left(\frac{a}{b}\right), R\left(-\frac{a}{b}\right)\right) \leq \begin{cases}\frac{39}{12-40 \lambda}, & \text { if } \lambda<\frac{21}{187} \\ \frac{47}{15-53 \lambda}, & \text { if } \frac{21}{187} \leq \lambda<\frac{3}{13} .\end{cases}
$$

\subsection{A degree 2 Mahler function}

As an example of degree 2 Mahler functions we take the function $S(z)$ satisfying $S(0)=1$ and

$$
z S(z)-\left(1+z+z^{2}\right) S\left(z^{4}\right)+S\left(z^{16}\right)=0 .
$$

This function was introduced by Dilcher and Stolarsky [10, and it has been studied recently in several works, see e.g. [1], 3] and [8], in particular the algebraic independence of $S(\alpha), S^{\prime}(\alpha)$, $S\left(\alpha^{4}\right)$ and $S^{\prime}\left(\alpha^{4}\right)$ is proved in [3] for all algebraic $\alpha, 0<|\alpha|<1$. Note also that in [8] an upper bounded 5 is obtained for the irrationality exponent of $S(1 / b)$.

Theorem 5. We have

$$
\mu\left(S\left(\frac{1}{b}\right), S\left(\frac{1}{b^{4}}\right)\right) \leq \frac{167}{25}=6.68 .
$$

More generally, if $0 \leq \lambda<1 / 5$, then

$$
\mu\left(S\left(\frac{a}{b}\right), S\left(\left(\frac{a}{b}\right)^{4}\right)\right) \leq \frac{167}{25-93 \lambda} .
$$


All results above are based on non-vanishing of the determinants of suitable Hermite-Padé approximation polynomials studied in Section 2. This non-vanishing is verified here by computing the determinants, but it would be of great interest to find a more general criterion for this. After having some non-zero determinants the functional equations can be used to produce a sufficiently dense infinite sequence of approximations with non-zero determinants. It is wellknown that such approximations can be used to produce linear independence measures. Section 3 contains this consideration, and it is then applied to prove a general result in Section 4 . The proofs of Theorems 1-5 are given in Section 5.

\section{Important determinants}

We first note that the above system (1) can be given in the form

$$
\begin{aligned}
& P(z) F\left(z^{d}\right)=P_{11}(z) F(z)+P_{12}(z) G(z)+P_{10}(z), \\
& P(z) G\left(z^{d}\right)=P_{21}(z) F(z)+P_{22}(z) G(z)+P_{20}(z),
\end{aligned}
$$

where $P(z)$, the least common denominator of $p_{i j}(z)$, and $P_{i j}(z)=P(z) p_{i j}(z)$ belong to $\mathbb{Z}[z]$ and satisfy $P_{11}(z) P_{22}(z)-P_{12}(z) P_{21}(z) \neq 0$.

For an integer $k \geq 1$, let $A_{k}(z), B_{k}(z), C_{k}(z) \in \mathbb{Z}[z]$ denote $\left(d_{1}, d_{2}, d_{3}\right)=\left(d_{1}(k), d_{2}(k), d_{3}(k)\right)$ Hermite-Padé approximation polynomials of $F(z), G(z)$ and 1 , so

$$
A_{k}(z) F(z)+B_{k}(z) G(z)+C_{k}(z)=R_{k}(z)
$$

where $\operatorname{deg} A_{k}(z) \leq d_{1}, \operatorname{deg} B_{k}(z) \leq d_{2}$, deg $C_{k}(z) \leq d_{3}$ and the order of zero at $z=0$ of the remainder term $R_{k}(z)$ satisfies ord $R_{k}(z)=: o(k) \geq d_{1}+d_{2}+d_{3}+2$. Clearly such polynomials, where at least one of $A_{k}(z), B_{k}(z)$ is not zero, exist. Substituting in (10) $z^{d}$ for $z$ and applying (8) and (9), we obtain

$$
\begin{aligned}
\left(P_{11}(z) A_{k}\left(z^{d}\right)\right. & \left.+P_{21}(z) B_{k}\left(z^{d}\right)\right) F(z)+\left(P_{12}(z) A_{k}\left(z^{d}\right)+P_{22}(z) B_{k}\left(z^{d}\right)\right) G(z) \\
& +P_{10}(z) A_{k}\left(z^{d}\right)+P_{20}(z) B_{k}\left(z^{d}\right)+P(z) C_{k}\left(z^{d}\right)=P(z) R_{k}\left(z^{d}\right) .
\end{aligned}
$$

Repeating this procedure $m$ times, we have

$$
A_{k, m}(z) F(z)+B_{k, m}(z) G(z)+C_{k, m}(z)=R_{k, m}(z), m=0,1, \ldots,
$$

where $A_{k, 0}(z)=A_{k}(z), B_{k, 0}(z)=B_{k}(z), C_{k, 0}(z)=C_{k}(z), R_{k, 0}(z)=R_{k}(z)$ and, for $m=$ $1,2, \ldots$,

$$
\left\{\begin{aligned}
A_{k, m}(z)= & P_{11}(z) A_{k, m-1}\left(z^{d}\right)+P_{21}(z) B_{k, m-1}\left(z^{d}\right), \\
B_{k, m}(z)= & P_{12}(z) A_{k, m-1}\left(z^{d}\right)+P_{22}(z) B_{k, m-1}\left(z^{d}\right), \\
C_{k, m}(z)= & P_{10}(z) A_{k, m-1}\left(z^{d}\right)+P_{20}(z) B_{k, m-1}\left(z^{d}\right) \\
& +P(z) C_{k, m-1}\left(z^{d}\right), \\
R_{k, m}(z)= & P(z) R_{k, m-1}\left(z^{d}\right) .
\end{aligned}\right.
$$

We are interested in determinants

$$
\Delta(\underline{k}, m, z):=\operatorname{det}\left(\begin{array}{ccc}
A_{k_{1}, m}(z) & B_{k_{1}, m}(z) & C_{k_{1}, m}(z) \\
A_{k_{2}, m}(z) & B_{k_{2}, m}(z) & C_{k_{2}, m}(z) \\
A_{k_{3}, m}(z) & B_{k_{3}, m}(z) & C_{k_{3}, m}(z)
\end{array}\right),
$$

where $1 \leq k_{1}<k_{2}<k_{3}$. By the above recursions (12)

$$
\Delta(\underline{k}, m, z)=\Phi(z) \Delta\left(\underline{k}, m-1, z^{d}\right), \quad \Phi(z):=\left(P_{11}(z) P_{22}(z)-P_{12}(z) P_{21}(z)\right) P(z),
$$


and so

$$
\Delta(\underline{k}, m, z)=\Delta\left(\underline{k}, 0, z^{d^{m}}\right) \prod_{j=0}^{m-1} \Phi\left(z^{d^{j}}\right) .
$$

In particular, for degree one functions we have $\Phi(z)=P_{11}(z) P_{22}(z) P(z)$, and for degree two function $F(z)$ with $G(z)=F\left(z^{d}\right)$ the function $\Phi(z)=P_{21}(z) P^{2}(z)$.

Let $\bar{d}(k):=\max \left\{d_{1}(k), d_{2}(k), d_{3}(k)\right\}$. By our assumption $k_{1}<k_{2}<k_{3}$ it is natural to assume that $\bar{d}\left(k_{1}\right) \leq \bar{d}\left(k_{2}\right) \leq \bar{d}\left(k_{3}\right)$ and $o\left(k_{1}\right) \leq o\left(k_{2}\right) \leq o\left(k_{3}\right)$. Since

$$
\Delta(\underline{k}, 0, z)=\operatorname{det}\left(\begin{array}{ccc}
A_{k_{1}}(z) & B_{k_{1}}(z) & R_{k_{1}}(z) \\
A_{k_{2}}(z) & B_{k_{2}}(z) & R_{k_{2}}(z) \\
A_{k_{3}}(z) & B_{k_{3}}(z) & R_{k_{3}}(z)
\end{array}\right),
$$

it follows that $o\left(k_{1}\right) \leq \operatorname{ord} \Delta(\underline{k}, 0, z) \leq \operatorname{deg} \Delta(\underline{k}, 0, z) \leq \bar{d}\left(k_{1}\right)+\bar{d}\left(k_{2}\right)+\bar{d}\left(k_{3}\right)$, if $\Delta(\underline{k}, 0, z) \neq 0$. Thus in this case

$$
\Delta(\underline{k}, 0, z)=: z^{o\left(k_{1}\right)} D(\underline{k}, z)
$$

with some polynomial $D(\underline{k}, z) \neq 0, \operatorname{deg} D(\underline{k}, z) \leq \bar{d}\left(k_{1}\right)+\bar{d}\left(k_{2}\right)+\bar{d}\left(k_{3}\right)-o\left(k_{1}\right)$. Further, if $o\left(k_{1}\right)>\bar{d}\left(k_{1}\right)+\bar{d}\left(k_{2}\right)+\bar{d}\left(k_{3}\right)$, then $\Delta(\underline{k}, 0, z)=0$.

We note that the condition $D(\underline{k}, z) \neq 0$ gives a strong restriction to $o\left(k_{1}\right)$. For example, if $d_{j}\left(k_{1}\right)=k, d_{j}\left(k_{2}\right)=k+1, d_{j}\left(k_{3}\right)=k+2(j=1,2,3)$, then $\operatorname{deg} \Delta(\underline{k}, 0, z) \leq 3 k+3$ and $o\left(k_{1}\right) \geq 3 k+2$. Thus the condition $D(\underline{k}, z) \neq 0$ is possible only if $3 k+2 \leq o\left(k_{1}\right) \leq 3 k+3$.

The above means that one determinant $\Delta(\underline{k}, 0, z) \neq 0$ gives an infinite sequence of determinants $\Delta(\underline{k}, m, z) \neq 0, m=0,1, \ldots$. When considering the values of the functions at rational points $z=a / b$ we need to know that $\Delta(\underline{k}, m, a / b) \neq 0$ at least for all sufficiently large $m$. This condition can be verified in many concrete cases by using (13) and (14), since $\operatorname{deg} D(\underline{k}, z)$ is small.

\section{Fundamental lemma}

In this section $\gamma_{1}$ and $\gamma_{2}$ denote real numbers and $b \geq 2$ is an integer. Let $\underline{k}=\underline{k}(\ell)=$ $\left(k_{\ell, 1}, k_{\ell, 2}, k_{\ell, 3}\right)(\ell=1, \ldots, L)$ be vectors with positive integer components $k_{\ell, i}$ satisfying $k_{\ell, 1}<$ $k_{\ell, 2}<k_{\ell, 3}$ and $k_{\ell, 3} \leq k_{\ell+1,1}(\ell=1, \ldots, L-1), k_{L, 3} \leq d k_{1,1}$. Assume that for each $k=k_{\ell, i}$ there exists an integer $m_{0}(k)$ such that for all $m \geq m_{0}(k)$ we have linear forms

$$
a_{k, m} \gamma_{1}+b_{k, m} \gamma_{2}+c_{k, m}=r_{k, m}
$$

with the following properties (i) - (iii).

(i) The coefficients $a_{k, m}, b_{k, m}, c_{k, m} \in \mathbb{Z}$ and satisfy

$$
\max \left\{\left|a_{k, m}\right|,\left|b_{k, m}\right|\right\} \leq c_{1}(k) b^{E(k) d^{m}},
$$

where $E(k)$ and $c_{1}(k)$ (as also $c_{2}(k), \ldots$ later) are positive constants independent of $m$.

(ii) We have

$$
\left|r_{k, m}\right| \leq c_{2}(k) b^{-V(k) d^{m}}
$$

where $V(k)>0$ is independent of $m$.

(iii) The determinant

$$
\operatorname{det}\left(\begin{array}{lll}
a_{k_{\ell, 1}, m} & b_{k_{\ell, 1}, m} & c_{k_{\ell, 1}, m} \\
a_{k_{\ell, 2}, m} & b_{k_{\ell, 2}, m} & c_{k_{\ell, 2}, m} \\
a_{k_{\ell, 3}, m} & b_{k_{\ell, 3}, m} & c_{k_{\ell, 3}, m}
\end{array}\right) \neq 0 .
$$

for all $\ell=1, \ldots, L ; m \geq m_{0}(\underline{k}(\ell))=\max _{1 \leq i \leq 3}\left\{m_{0}\left(k_{\ell, i}\right)\right\}$. 
For the following fundamental lemma, we finally define, for all $\ell=1, \ldots, L$, the notations

$$
\begin{aligned}
& \theta(\ell)=\max _{1 \leq i<j \leq 3}\left\{E\left(k_{\ell, i}\right)+E\left(k_{\ell, j}\right)\right\}, \\
& \nu(\ell)=\min _{\substack{1 \leq i, j \leq 3 \\
i \neq j}}\left\{V\left(k_{\ell, i}\right)-E\left(k_{\ell, j}\right)\right\},
\end{aligned}
$$

and denote $K:=(\underline{k}(1), \ldots, \underline{k}(L))$.

Lemma 1. Suppose that $0<\nu(1)<\cdots<\nu(L)<d \nu(1)$. Then there exist positive constants $C=C(K)$ and $H_{0}=H_{0}(K)$ such that for any integers $h_{0}, h_{1}, h_{2}$, not all zero,

$$
\left|h_{0}+h_{1} \gamma_{1}+h_{2} \gamma_{2}\right|>C H^{-\mu}
$$

where $H=\max \left\{\left|h_{1}\right|,\left|h_{2}\right|, H_{0}\right\}$ and

$$
\mu=\max _{1 \leq \ell \leq L} \mu(\ell), \quad \mu(\ell):=\frac{\theta(\ell+1)}{\nu(\ell)}, \quad \theta(L+1):=d \theta(1) .
$$

Proof. Let

$$
\Lambda=h_{0}+h_{1} \gamma_{1}+h_{2} \gamma_{2} .
$$

By the condition (iii) above, for all $\ell=1, \ldots, L$ there exist $1 \leq i<j \leq 3$ such that

$$
D(\underline{k}(\ell), \underline{h}):=\operatorname{det}\left(\begin{array}{ccc}
h_{1} & h_{2} & h_{0} \\
a_{k_{\ell, i}, m} & b_{k_{\ell, i}, m} & c_{k_{\ell, i}, m} \\
a_{k_{\ell, j}, m} & b_{k_{\ell, j}, m} & c_{k_{\ell, j}, m}
\end{array}\right)=\operatorname{det}\left(\begin{array}{ccc}
h_{1} & h_{2} & \Lambda \\
a_{k_{\ell, i}, m} & b_{k_{\ell, i}, m} & r_{k_{\ell, i}, m} \\
a_{k_{\ell, j}, m} & b_{k_{\ell, j}, m} & r_{k_{\ell, j}, m}
\end{array}\right) \neq 0 .
$$

Since $D(\underline{k}(\ell), \underline{h})$ is an integer, we obtain, by (15) and (16),

$$
\begin{gathered}
1 \leq 2|\Lambda| c_{1}\left(k_{\ell, i}\right) c_{1}\left(k_{\ell, j}\right) b^{\left(E\left(k_{\ell, i}\right)+E\left(k_{\ell, j}\right)\right) d^{m}}+2 h c_{1}\left(k_{\ell, j}\right) c_{2}\left(k_{\ell, i}\right) b^{-\left(V\left(k_{\ell, i}\right)-E\left(k_{\ell, j}\right)\right) d^{m}}+ \\
2 h c_{1}\left(k_{\ell, i}\right) c_{2}\left(k_{\ell, j}\right) b^{-\left(V\left(k_{\ell, j}\right)-E\left(k_{\ell, i}\right)\right) d^{m}}
\end{gathered}
$$

with $h=\max \left\{\left|h_{1}\right|,\left|h_{2}\right|\right\}$. The definitions of $\theta(\ell)$ and $\nu(\ell)$ then give

$$
1 \leq C_{1}(K)|\Lambda| b^{\theta(\ell) d^{m}}+C_{2}(K) h b^{-\nu(\ell) d^{m}}
$$

for all $m \geq M_{0}:=\max \left\{m_{0}(\underline{k}(1)), \ldots, m_{0}(\underline{k}(L))\right\}$, and here $C_{1}(K)$ and $C_{2}(K)$ (and also $C_{3}(K)$ later) are positive constants depending on $K$. Note that $C_{1}(K)$ and $C_{2}(K)$ are the same for all $\ell$.

We now choose $H_{0}$ in such a way that

$$
2 C_{2}(K) H_{0} \geq b^{\nu(1) d^{M_{0}}},
$$

and fix the pair $(\ell, m)$ from the sequence $\left(1, M_{0}\right), \ldots,\left(L, M_{0}\right),\left(1, M_{0}+1\right), \ldots,\left(L, M_{0}+1\right)$, $\left(1, M_{0}+2\right), \ldots$ to be the first one satisfying

$$
2 C_{2}(K) H<b^{\nu(\ell) d^{m}}
$$

where $H=\max \left\{h, H_{0}\right\}$. Then $(\ell, m) \neq\left(1, M_{0}\right)$, and the pair just before it is $(\ell-1, m)$, if $\ell>1$, and $(L, m-1)$, if $\ell=1$. The above choice means that

$$
\begin{aligned}
& 2 C_{2}(K) H \geq b^{\nu(\ell-1) d^{m}}, \quad \ell>1, \\
& 2 C_{2}(K) H \geq b^{\nu(L) d^{m-1}}, \quad \ell=1 .
\end{aligned}
$$


In the first case, by (18),

$$
\frac{1}{2}<C_{1}(K)|\Lambda| b^{\theta(\ell) d^{m}}=C_{1}(K)|\Lambda|\left(b^{\nu(\ell-1) d^{m}}\right)^{\theta(\ell) / \nu(\ell-1)} \leq C_{3}(K)|\Lambda| H^{\mu} .
$$

In the case $\ell=1$ we similarly have

$$
\frac{1}{2}<C_{1}(K)|\Lambda| b^{\theta(1) d^{m}}=C_{1}(K)|\Lambda|\left(b^{\nu(L) d^{m-1}}\right)^{d \theta(1) / \nu(L)} \leq C_{3}(K)|\Lambda| H^{\mu} .
$$

This proves our lemma.

\section{General theorem}

We now assume that $F(z), G(z) \in \mathbb{Q}[[z]]$ converge in some disk $D_{r}$ and satisfy (요 ) and (9). Our aim is to apply Lemma 1 to consider the function values $F(a / b)$ and $G(a / b)$ at non-zero rational points $a / b \in D_{r}$, where $\log |a|=\lambda \log b, 0 \leq \lambda<\log (r b) / \log b$. We also assume that

$$
\left(P_{11}\left((a / b)^{d^{j}}\right) P_{22}\left((a / b)^{d^{j}}\right)-P_{12}\left((a / b)^{d^{j}}\right) P_{21}\left((a / b)^{d^{j}}\right)\right) P\left((a / b)^{d^{j}}\right) \neq 0, j=0,1, \ldots
$$

The approximation forms we use are obtained from (11) at $z=a / b$. The recursions (12) imply, for all $m \geq 1$,

$$
\operatorname{deg} A_{k, m}(z), \operatorname{deg} B_{k, m}(z), \operatorname{deg} C_{k, m}(z) \leq\left(\bar{e}(k)+\frac{\tau}{d-1}\right) \cdot d^{m}-\frac{\tau}{d-1}
$$

where $\bar{e}(k)$ and $\tau$ are non-negative integers satisfying $\bar{e}(k) \leq \bar{d}(k):=\max \left\{d_{1}(k), d_{2}(k), d_{3}(k)\right\}$ and $\tau \leq \nu$, the maximum of the degrees of $P_{i j}(z)$ and $P(z)$. Thus the multiplication of (11) at $z=a / b$ by

$$
Q_{k, m}:=b^{\left(\bar{e}(k)+\frac{\tau}{d-1}\right) d^{m}-\frac{\tau}{d-1}}
$$

leads to linear forms

$$
a_{k, m} F\left(\frac{a}{b}\right)+b_{k, m} G\left(\frac{a}{b}\right)+c_{k, m}=r_{k, m}, \quad m=0,1, \ldots,
$$

where all $a_{k, m}, b_{k, m}$ and $c_{k, m}$ are integers. To be able to apply Lemma 1 with $\gamma_{1}=F(a / b), \gamma_{2}=$ $G(a / b)$ we need to estimate the coefficients $a_{k, m}$ and $b_{k, m}$ and the remainders $r_{k, m}$. For this we apply the recursions (12).

Let $\widetilde{P}(z)$ denote the polynomial, where the coefficient of $z^{j}$ is the maximum of the absolute values of the corresponding coefficients in $P_{i j}(z), 1 \leq i, j \leq 2$. Then, for all $m=1,2, \ldots$,

$$
\begin{aligned}
& \left|A_{k, m}(z)\right| \leq \widetilde{P}(|z|)\left(\left|A_{k, m-1}\left(z^{d}\right)\right|+\delta\left|B_{k, m-1}\left(z^{d}\right)\right|\right), \\
& \left|B_{k, m}(z)\right| \leq \widetilde{P}(|z|)\left(\delta\left|A_{k, m-1}\left(z^{d}\right)\right|+\left|B_{k, m-1}\left(z^{d}\right)\right|\right),
\end{aligned}
$$

where $\delta=0$ for degree one functions $F(z)$ and $G(z)$, and $\delta=1$ otherwise. Applying these inequalities we obtain

$$
\max \left\{\left|A_{k, m}(z)\right|,\left|B_{k, m}(z)\right|\right\} \leq(1+\delta)^{m} \max \left\{\left|A_{k}\left(z^{d^{m}}\right)\right|,\left|B_{k}\left(z^{d^{m}}\right)\right|\right\} \prod_{j=0}^{m-1} \widetilde{P}\left(|z|^{d^{j}}\right) .
$$

Therefore, for all $m \geq m_{1}(k)$,

$$
\max \left\{\left|a_{k, m}\right|,\left|b_{k, m}\right|\right\} \leq c_{3}(k) b^{\left(\bar{e}(k)+\frac{\tau}{d-1}\right) d^{m}},
$$


if the condition

$$
(1+\delta)|\widetilde{P}(0)| \leq 1
$$

holds. Generally, for any given $\delta_{1}>0$,

$$
\max \left\{\left|a_{k, m}\right|,\left|b_{k, m}\right|\right\} \leq c_{3}(k) b^{\left(\bar{e}(k)+\frac{\tau}{d-1}+\delta_{1}\right) d^{m}}
$$

for all $m \geq m_{2}\left(k, \delta_{1}\right)$, and under the condition (21) we may choose here $\delta_{1}=0$.

Since

$$
R_{k, m}(z)=R_{k}\left(z^{d^{m}}\right) \prod_{j=0}^{m-1} P\left(z^{d^{j}}\right)
$$

we also have

$$
\left|r_{k, m}\right| \leq c_{4}(k) \max \left\{1,|P(0)|^{m}\right\} b^{-\left((1-\lambda) o(k)-\bar{e}(k)-\frac{\tau}{d-1}\right) d^{m}}
$$

for all $m \geq m_{3}(k)$. Thus, for any given $\delta_{2}>0$,

$$
\left|r_{k, m}\right| \leq c_{4}(k) b^{-\left((1-\lambda) o(k)-\bar{e}(k)-\frac{\tau}{d-1}-\delta_{2}\right) d^{m}}
$$

for all $m \geq m_{4}\left(k, \delta_{2}\right)$, and we may use here the value $\delta_{2}=0$, if the condition

$$
|P(0)| \leq 1
$$

holds.

Thus we have the estimates (15) and (16) for all $m \geq m_{5}\left(k, \delta_{1}, \delta_{2}\right)$, where

$$
E(k)=\bar{e}(k)+\frac{\tau}{d-1}+\delta_{1}, V(k)=(1-\lambda) o(k)-\bar{e}(k)-\frac{\tau}{d-1}-\delta_{2} .
$$

By using these values with Lemma 1 we get the following theorem, we only need to note that the condition $D(\underline{k}, z) \neq 0$ implies $D\left(\underline{k},(a / b)^{d^{m}}\right) \neq 0$ for all $m \geq m_{6}(\underline{k}, a / b)$.

Theorem 6. Assume that the condition (19) holds and $D(\underline{k}, z) \neq 0$ for all $\ell=1, \ldots, L$. Let $\theta(\ell)$ and $\nu(\ell)$ be defined as in Lemma 1 with $E(k)$ and $V(k)$ given in (25). If $0<\nu(1)<\cdots<$ $\nu(L)<d \nu(1)$, then there exist positive constants $\lambda_{0}=\lambda_{0}(K, F, G), C=C(K, a / b, F, G)$ and $H_{0}=H_{0}(K, a / b, F, G)$ such that for all $0 \leq \lambda<\lambda_{0}$ and any integers $h_{0}, h_{1}, h_{2}$, not all zero,

$$
\left|h_{0}+h_{1} F\left(\frac{a}{b}\right)+h_{2} G\left(\frac{a}{b}\right)\right|>C H^{-\mu}
$$

with $H$ and $\mu$ as in Lemma 1,

\section{Proof of Theorems 1-5}

We are ready to prove Theorems 1 15. We start by giving the following formulae which follow from (25):

$$
\left\{\begin{array}{l}
\theta(\ell)=\max _{1 \leq i<j \leq 3}\left\{\bar{e}\left(k_{\ell, i}\right)+\bar{e}\left(k_{\ell, j}\right)\right\}+\frac{2 \tau}{d-1}+2 \delta_{1}, \\
\nu(\ell)=\min _{\substack{1 \leq i, j \leq 3 \\
i \neq j}}\left\{(1-\lambda) o\left(k_{\ell, i}\right)-\bar{e}\left(k_{\ell, i}\right)-\bar{e}\left(k_{\ell, j}\right)\right\}-\frac{2 \tau}{d-1}-\delta_{1}-\delta_{2} .
\end{array}\right.
$$

Thus we should choose $\tau$ and $\delta_{i}$ as small as possible while applying Lemma 1, 
Proof of Theorem 1. To prove Theorem 1, we apply Theorem [6 with $F(z)=T(z), G(z)=$ $T^{2}(z)$. Now, by (3),

$$
(1-z)^{2} F\left(z^{2}\right)=(1-z) F(z), \quad(1-z)^{2} G\left(z^{2}\right)=G(z) .
$$

Therefore $r=1, \delta=0, P(z)=(1-z)^{2}, \widetilde{P}(z)=1+z$ and $\widetilde{P}(0)=P(0)=1$ gives $\delta_{1}=\delta_{2}=0$. We shall use $(k, k+1, k-1)$ approximations and we may take $\bar{e}(k)=k+1, \tau=0$. Our $\underline{k}(\ell)$ are $\left(k_{\ell, 1}, k_{\ell, 1}+1, k_{\ell, 1}+2\right)$ and the choices for $k=k_{\ell, 1}$ are 29,31,34,43 and 49. For all these values $o(k)=3 k+2$. Since $\operatorname{deg} \Delta(\underline{k}(\ell), z) \leq 3 k+3$, we have $D(\underline{k}(\ell), z)=s_{\ell, 0}+s_{\ell, 1} z$, where

$$
s_{\ell, 0}=\operatorname{det}\left(\begin{array}{ll}
A_{k+1}(0) & B_{k+1}(0) \\
A_{k+2}(0) & B_{k+2}(0)
\end{array}\right) c \neq 0
$$

where $c$ is the coefficient of $z^{3 k+2}$ in $R_{k}(z)$ (see Appendix). In fact $s_{\ell, 0}$ is nonzero in all of our cases, also in the proofs of Theorems 225. By using (26) we get

$$
\theta(\ell)=2 k+5, \quad \nu(\ell)=k-2-\lambda(3 k+2)
$$

for all $\lambda<2 / 3$. So we have the following table.

\begin{tabular}{|c|c|c|c|c|c|}
\hline$\ell$ & 1 & 2 & 3 & 4 & 5 \\
\hline$k$ & 29 & 31 & 34 & 43 & 49 \\
\hline$\theta(\ell)$ & 63 & 67 & 73 & 91 & 103 \\
\hline$\nu(\ell)$ & $27-89 \lambda$ & $29-95 \lambda$ & $32-104 \lambda$ & $41-131 \lambda$ & $47-149 \lambda$ \\
\hline
\end{tabular}

For the condition $0<\nu(1)<\cdots<\nu(5)<2 \nu(1)$ we need to assume $\lambda<\lambda_{0}:=7 / 29 \approx 0.241 \ldots$ When $\lambda<\lambda_{0}$, the comparison of $\mu(\ell)$ gives

$$
\mu=\max _{\ell} \frac{\theta(\ell+1)}{\nu(\ell)}=\frac{\theta(4)}{\nu(3)}=\frac{91}{32-104 \lambda} .
$$

This prove Theorem 1 .

To prove Theorem 2 [5, we need to modify the choices of parameters.

Proof of Theorem [. Here we apply Theorem 6 with $F(z)=A(z), G(z)=B(z)$, and the use of (4) gives $r=1, \delta=0, P(z)=1+z+z^{2}, \widetilde{P}(z)=1$ and $\delta_{1}=\delta_{2}=0$. The $(k, k+1, k-1)$ approximations give $\bar{e}(k)=k+1, \tau=1$. By choosing $\underline{k}(l)$ as above, where $k=k_{\ell, 1}$ are 29, 31, $34,38,43$ and 49 , we get $o(k)=3 k+2$ and the determinants $D(\underline{k}(\ell), z) \neq 0$ (see Appendix). Further,

$$
\theta(\ell)=2 k+7, \quad \nu(\ell)=k-4-\lambda(3 k+2)
$$

for all $\lambda<2 / 3$, and this leads to the following table.

\begin{tabular}{|c|c|c|c|c|c|c|}
\hline$\ell$ & 1 & 2 & 3 & 4 & 5 & 6 \\
\hline$k$ & 29 & 31 & 34 & 38 & 43 & 49 \\
\hline$\theta(\ell)$ & 65 & 69 & 75 & 83 & 93 & 105 \\
\hline$\nu(\ell)$ & $25-89 \lambda$ & $27-95 \lambda$ & $30-104 \lambda$ & $34-116 \lambda$ & $39-131 \lambda$ & $45-149 \lambda$ \\
\hline
\end{tabular}

To satisfy the condition $0<\nu(1)<\cdots<\nu(6)<2 \nu(1)$, we need to assume $\lambda<\lambda_{0}:=5 / 29 \approx$ $0.172 \ldots$ After the comparison of $\mu(\ell)=\theta(\ell+1) / \nu(\ell)$ we see that

$$
\mu=\max _{1 \leq \ell \leq 6} \mu(\ell)= \begin{cases}\mu(6)=\frac{130}{45-149 \lambda}, & \text { if } \lambda<\frac{145}{1289}, \\ \mu(1)=\frac{69}{25-89 \lambda}, & \text { if } \frac{145}{1289} \leq \lambda<\frac{5}{29} .\end{cases}
$$

This proves Theorem 2 , 
Remark 1. We note that here all determinants $D(\underline{k}(\ell), z) \neq 0,1 \leq k \leq 50$. In all other theorems most of these determinants equal zero.

Proof of Theorem [3. In this case we apply Theorem 6 with $d=3, F(z)=G_{3}(z)$ and $G(z)=$ $F_{3}(z)$. Then (5) implies $r=1, \delta=0, P(z)=1-z^{2}, \widetilde{P}(z)=1+z^{2}$ and $\delta_{1}=\delta_{2}=0$. The use of $(k, k, k)$ approximations give $\bar{e}(k)=k, \tau=2$. If $\underline{k}(\ell)$ is the same as above and $k=k_{\ell, 1}$ are 19, 26 and 39, then $o(k)=3 k+2$ and $D(\underline{k}(\ell), z) \neq 0$ (see Appendix). By (26) $)$, if $\lambda<2 / 3$, we get

$$
\theta(\ell)=2 k+5, \quad \nu(\ell)=k-2-\lambda(3 k+2) .
$$

Now the table is the following.

\begin{tabular}{|c|c|c|c|}
\hline$\ell$ & 1 & 2 & 3 \\
\hline$k$ & 19 & 26 & 39 \\
\hline$\theta(\ell)$ & 43 & 57 & 83 \\
\hline$\nu(\ell)$ & $17-59 \lambda$ & $24-80 \lambda$ & $37-119 \lambda$ \\
\hline
\end{tabular}

The condition $0<\nu(1)<\nu(2)<\nu(3)<3 \nu(1)$ holds, if $\lambda<\lambda_{0}:=7 / 29 \approx 0.241 \ldots$ Similarly to the above proofs we now get Theorem 3 ,

Proof of Theorem 4. Here we may use Theorem [6 with $F(z)=R(z)$ and $G(z)=R(-z)$. By (6)), we have

$$
2 z F\left(z^{2}\right)=z F(z)+z G(z), \quad 2 z G\left(z^{2}\right)=F(z)+G(z) .
$$

Therefore, we can choose $r=1, \delta=1, P(z)=2 z$, and $\widetilde{P}(z)=1+z$. Since $P(0)=0,(23)$ holds and we may take $\delta_{2}=0$. We use the $(k, k, k)$ approximations and we can take $\bar{e}(k)=k$ and $\tau=1$. We also choose $\underline{k}(\ell)=\left(k_{\ell, 1}, k_{\ell, 1}+1, k_{\ell, 1}+2\right)$ where $k=k_{\ell, 1}$ are 17,21 and 26 . Then we get $o(k)=3 k+2$ and the determinants $D(\underline{k}(\ell), z) \neq 0$ (see Appendix). Moreover,

$$
\theta(\ell)=2 k+5+2 \delta_{1}, \quad \nu(\ell)=k-2-\delta_{1}-\lambda(3 k+2)
$$

for all $\lambda<2 / 3$. This gives the following table.

\begin{tabular}{|c|c|c|c|}
\hline$\ell$ & 1 & 2 & 3 \\
\hline$k$ & 17 & 21 & 26 \\
\hline$\theta(\ell)$ & $39+2 \delta_{1}$ & $47+2 \delta_{1}$ & $57+2 \delta_{1}$ \\
\hline$\nu(\ell)$ & $15-\delta_{1}-53 \lambda$ & $19-\delta_{1}-65 \lambda$ & $24-\delta_{1}-80 \lambda$ \\
\hline
\end{tabular}

The condition $0<\nu(1)<\nu(2)<\nu(3)<2 \nu(1)$ holds, if $\lambda<3 / 13 \approx 0.230 \ldots$ and $\delta_{1}$ is sufficiently small. If $\lambda<21 / 187 \approx 0.112 \ldots$ and $\delta_{1}$ is small enough, then

$$
\mu=\frac{\theta(4)}{\nu(3)}=\frac{78+4 \delta_{1}}{24-\delta_{1}-80 \lambda}
$$

If $21 / 187 \leq \lambda<3 / 13$, then

$$
\mu=\frac{\theta(2)}{\nu(1)}=\frac{47+2 \delta_{1}}{15-\delta_{1}-53 \lambda} .
$$

This proves Theorem 4, since we may choose $\delta_{1}$ arbitrarily small.

Proof of Theorem [5. We now apply Theorem 6 with $F(z)=S(z)$ and $G(z)=S\left(z^{4}\right)$. The use of (77) gives $d=4, r=1$ and

$$
F\left(z^{4}\right)=G(z), \quad G\left(z^{4}\right)=-z F(z)+\left(1+z+z^{2}\right) G(z) .
$$


Since $P(0)=1$, we may choose $\delta_{2}=0$ in (23). We shall use of $(k, k-1, k)$ approximations and we may take $\bar{e}(k)=k, \tau=1$. Again our $\underline{k}(\ell)=\left(k_{\ell, 1}, k_{\ell, 1}+1, k_{\ell, 1}+2\right)$ and the choices for $k=k_{\ell, 1}$ are 10 and 26. For both of these values $o(k)=3 k+1$ and the determinants $D(\underline{k}(\ell), z) \neq 0$ (see Appendix). By using (26), if $\lambda<2 / 3$, we get,

$$
\theta(\ell)=2 k+3+\frac{2}{3}+2 \delta_{1}, \quad \nu(\ell)=k-1-\frac{2}{3}-\delta_{1}-\lambda(3 k+1) .
$$

Thus we have the following table.

\begin{tabular}{|c|c|c|}
\hline$\ell$ & 1 & 2 \\
\hline$k$ & 10 & 26 \\
\hline$\theta(\ell)$ & $23+\frac{2}{3}+2 \delta_{1}$ & $55+\frac{2}{3}+2 \delta_{1}$ \\
\hline$\nu(\ell)$ & $9-\frac{2}{3}-\delta_{1}-31 \lambda$ & $25-\frac{2}{3}-\delta_{1}-79 \lambda$ \\
\hline
\end{tabular}

If $\lambda<\lambda_{0}:=\frac{1}{5}$ and $\delta_{1}>0$ is sufficiently small, then $0<\nu(1)<\nu(2)<4 \nu(1)$. Since

$$
\frac{55+\frac{2}{3}+2 \delta_{1}}{9-\frac{2}{3}-\delta_{1}-31 \lambda}>\frac{4\left(23+\frac{2}{3}+2 \delta_{1}\right)}{25-\frac{2}{3}-\delta_{1}-79 \lambda}
$$

for all $0 \leq \lambda<\lambda_{0}$, and $\delta_{1}>0$ can be arbitrarily small, Theorem 5 follows from Theorem 6 .

\section{References}

[1] Boris Adamczewski. Non-converging continued fractions related to the Stern diatomic sequence. Acta Arith., 142: 67-78, 2010.

[2] Boris Adamczewski and Tanguy Rivoal. Irrationality measures for some automatic real numbers. Math. Proc. Cambridge Phil. Soc., 147 (11): 659-678, 2009.

[3] Richard P. Brent, Michael Coons, and Wadim Zudilin. Algebraic independence of Mahler functions via radial asymptotics . Int. Math. Res. Notices, 2016 (2): 571-603, 2016.

[4] Yann Bugeaud. On the rational approximation to the Thue-Morse-Mahler numbers. Ann. Inst. Fourier (Grenoble), 61 (5): 2065-2076, 2011.

[5] Yann Bugeaud, Guo-Niu Han, Zhi-Ying Wen, and Jia-Yan Yao. Hankel determinants, Padé approximations, and irrationality exponents. Int. Math. Res. Notices, 2016 (5): 1467-1496, 2016.

[6] Peter Bundschuh and Keijo Väänänen. Transcendence results on the generating Lambert series of the powers of a fixed integer. Hardy-Ramanujan Journal, 38: 36-44, 2015.

[7] Peter Bundschuh and Keijo Väänänen. Algebraic independence of the generating functions of Stern's sequence and of its twist. J. Théor. Nombres Bordeaux, 25: 43-57, 2013.

[8] Peter Bundschuh and Keijo Väänänen. Transcendence results on the generating functions of the characteristic functions of certain self-generating sets. Acta Arith., 162: 273-288, 2014 .

[9] Michael Coons. On the rational approximation of the sum of the reciprocals of the Fermat numbers. Ramanujan J., 30 (1): 39-65, 2013.

[10] Karl Dilcher and Kenneth B. Stolarsky. Stern polynomials and double-limit continued fractions. Acta Arith., 140: 119-134, 2009. 
[11] Ying-Jun Guo, Zhi-Xiong Wen, and Wen Wu. On the irrationality exponent of the regular paperfolding numbers. Linear Algebra Appl., 446: 237 - 264, 2014.

[12] K. Nishioka, Mahler Functions and Transcendence, Lecture Notes in Math., vol. 1631, Springer, 1996.

[13] Keijo Väänänen. On rational approximations of certain Mahler functions with a connection to the Thue-Morse sequence. Int. J. Number Theory, 11 (2): 487-493, 2015.

[14] Keijo Väänänen and Wen Wu. On simultaneous approximation of the values of certain Mahler functions. arXiv:1505.00931,

[15] Zhi-Xiong Wen and Wen Wu. Hankel determinants of the Cantor sequence. Scientia Sinica Mathematica (Chinese), 44 (10): 1059-1072, 2014.

Keijo Väänänen

Department of Mathematical Sciences, University of Oulu,

P.O. Box 3000, 90014 Oulu, Finland.

keijo.vaananen@oulu.fi
Wen $\mathrm{Wu}$

Faculty of Mathematics and Statistics, Hubei University, 430062 Wuhan, P.R. China.

Department of Mathematical Sciences, University of Oulu, P.O. Box 3000, 90014 Oulu, Finland.

hust . wuwen@gmail. com

\section{Appendix}

In the appendix we shall give the values of $\Delta(\underline{k}, 0, z)$, where $\underline{k}=(k, k+1, k+2)$. Tables in this appendix are organized in the following form.

\begin{tabular}{l|l|l}
\hline \multirow{3}{*}{$k$} & \multirow{2}{*}{$o(k)$} & $A_{k}(z)$ \\
\cline { 3 - 3 } & & $B_{k}(z)$ \\
\cline { 3 - 3 } & & The first two non-zero terms of $R_{k}(z)$ \\
\hline \multirow{2}{*}{$\underline{k}$} & $\Delta(\underline{k}, 0, z)$ \\
\hline
\end{tabular}

\section{Approximation polynomials and related determinants in Theorem 1}

\begin{tabular}{|c|c|c|}
\hline \multirow{3}{*}{29} & \multirow{3}{*}{89} & $\begin{array}{l}-5500 z^{29}+264 z^{28}-432 z^{27}-432 z^{26}-5908 z^{25}-144 z^{24}-816 z^{23}-816 z^{22}- \\
6268 z^{21}-504 z^{20}-1152 z^{19}-1152 z^{18}-6580 z^{17}-816 z^{16}-1440 z^{15}-1440 z^{14}+ \\
4132 z^{13}-1632 z^{12}-864 z^{11}-864 z^{10}+4684 z^{9}-1080 z^{8}-336 z^{7}-336 z^{6}+25048 z^{5}+ \\
1992 z^{4}+1224 z^{3}+1224 z^{2}-18708 z-1416\end{array}$ \\
\hline & & $\begin{array}{l}8561 z^{30}+9917 z^{29}+18922 z^{28}+20722 z^{27}+24653 z^{26}+27143 z^{25}+31050 z^{24}+ \\
33516 z^{23}+36870 z^{22}+40224 z^{21}+43404 z^{20}+46584 z^{19}+42727 z^{18}+46075 z^{17}+ \\
41528 z^{16}+44186 z^{15}+43421 z^{14}+46979 z^{13}+45716 z^{12}+48776 z^{11}+40170 z^{10}+ \\
43092 z^{9}+33508 z^{8}+35452 z^{7}+27159 z^{6}+28953 z^{5}+19844 z^{4}+20822 z^{3}+10939 z^{2}+ \\
11143 z+528\end{array}$ \\
\hline & & $-57640 z^{89}+61912 z^{90}$ \\
\hline & & $\begin{array}{l}744 z^{30}-764 z^{28}+844 z^{26}-664 z^{24}+944 z^{22}-564 z^{20}+1044 z^{18}-464 z^{16}-444 z^{14}+ \\
1064 z^{12}-544 z^{10}+964 z^{8}+444 z^{6}-1064 z^{4}-1388 z^{2}+1628\end{array}$ \\
\hline
\end{tabular}




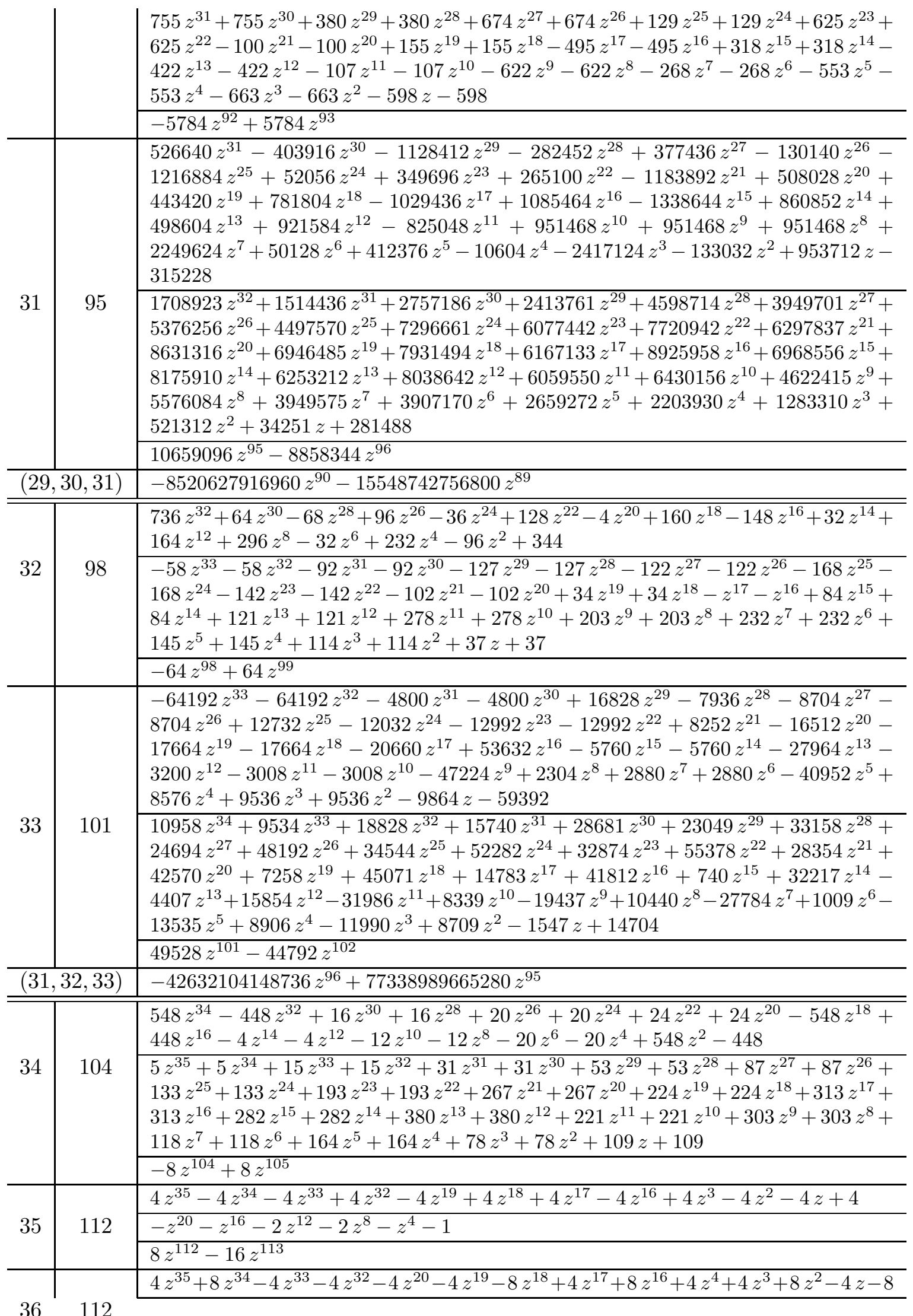




\begin{tabular}{|c|c|c|}
\hline & & $\begin{array}{l}z^{37}+z^{36}+2 z^{35}+2 z^{34}+3 z^{33}+3 z^{32}+4 z^{31}+4 z^{30}+6 z^{29}+6 z^{28}+8 z^{27}+8 z^{26}+ \\
10 z^{25}+10 z^{24}+12 z^{23}+12 z^{22}+15 z^{21}+14 z^{20}+15 z^{19}+15 z^{18}+17 z^{17}+16 z^{16}+ \\
16 z^{15}+16 z^{14}+18 z^{13}+16 z^{12}+14 z^{11}+14 z^{10}+14 z^{9}+12 z^{8}+8 z^{7}+8 z^{6}+8 z^{5}+ \\
7 z^{4}+5 z^{3}+5 z^{2}+4 z+3\end{array}$ \\
\hline & & $-16 z^{112}+8 z^{113}$ \\
\hline$(34$ & $5,36)$ & $32 z^{105}-32 z^{104}$ \\
\hline & & $4 z^{43}-8 z^{42}+8 z^{40}-8 z^{39}+8 z^{38}-8 z^{36}+4 z^{35}$ \\
\hline 43 & 131 & $-z^{44}+z^{43}-z^{36}+z^{35}-2 z^{28}+2 z^{27}-2 z^{20}+2 z^{19}-z^{12}+z^{11}-z^{4}+z^{3}$ \\
\hline & & $-8 z^{131}+24 z^{132}$ \\
\hline & & $64 z^{44}-88 z^{42}-44 z^{40}+88 z^{38}-4 z^{36}-4 z^{32}+4 z^{20}+4 z^{16}+4 z^{12}+4 z^{8}+4 z^{4}+4$ \\
\hline 44 & 134 & $\begin{array}{l}-15 z^{45}-15 z^{44}-8 z^{43}-8 z^{42}-4 z^{41}-4 z^{40}-13 z^{37}-13 z^{36}-4 z^{35}-4 z^{34}+z^{33}+ \\
z^{32}+6 z^{31}+6 z^{30}-22 z^{29}-22 z^{28}-6 z^{27}-6 z^{26}+2 z^{25}+2 z^{24}+10 z^{23}+10 z^{22}- \\
18 z^{21}-18 z^{20}-2 z^{19}-2 z^{18}+4 z^{17}+4 z^{16}+10 z^{15}+10 z^{14}-5 z^{13}-5 z^{12}+2 z^{11}+ \\
2 z^{10}+4 z^{9}+4 z^{8}+6 z^{7}+6 z^{6}-10 z^{5}-10 z^{4}-4 z^{3}-4 z^{2}-2 z-2\end{array}$ \\
\hline & & $-176 z^{134}+176 z^{135}$ \\
\hline & & $\begin{array}{l}304192 z^{45}+262504 z^{44}-72072 z^{43}-72072 z^{42}-40788 z^{41}+35640 z^{40}+146432 z^{39}+ \\
146432 z^{38}+84828 z^{37}+77880 z^{36}+128128 z^{35}+128128 z^{34}+69956 z^{33}+63008 z^{32}+ \\
116688 z^{31}+116688 z^{30}+106392 z^{29}+106392 z^{28}+96096 z^{27}+96096 z^{26}+85800 z^{25}+ \\
85800 z^{24}+75504 z^{23}+75504 z^{22}+109652 z^{21}+116600 z^{20}+38896 z^{19}+38896 z^{18}+ \\
69612 z^{17}+76560 z^{16}-4576 z^{15}-4576 z^{14}+53596 z^{13}+60544 z^{12}+6864 z^{11}+ \\
6864 z^{10}+61604 z^{9}+68552 z^{8}+11440 z^{7}+11440 z^{6}+62748 z^{5}+69696 z^{4}+9152 z^{3}+ \\
9152 z^{2}+57028 z+63976\end{array}$ \\
\hline 45 & 137 & $\begin{array}{l}-57215 z^{46}-102271 z^{45}-130028 z^{44}-145626 z^{43}-190986 z^{42}-229398 z^{41}- \\
260744 z^{40}-285142 z^{39}-385343 z^{38}-469911 z^{37}-525496 z^{36}-565448 z^{35}- \\
628583 z^{34}-683033 z^{33}-700980 z^{32}-710242 z^{31}-835054 z^{30}-932074 z^{29}- \\
949350 z^{28}-938834 z^{27}-966634 z^{26}-980538 z^{25}-927114 z^{24}-859794 z^{23}- \\
928550 z^{22}-969514 z^{21}-918722 z^{20}-840138 z^{19}-825524 z^{18}-800488 z^{17}- \\
715518 z^{16}-620126 z^{15}-616995 z^{14}-601705 z^{13}-531364 z^{12}-448864 z^{11}- \\
396040 z^{10}-339742 z^{9}-258032 z^{8}-172848 z^{7}-169960 z^{6}-156650 z^{5}-117726 z^{4}- \\
68380 z^{3}-58456 z^{2}-45058 z-24266\end{array}$ \\
\hline & & $180648 z^{137}+288392 z^{138}$ \\
\hline$(43$ & $4,45)$ & $247104 z^{132}-247104 z^{131}$ \\
\hline & & $\begin{array}{l}-30924 z^{49}+7224 z^{48}+8904 z^{47}+8904 z^{46}+74616 z^{45}-1680 z^{44}-1176 z^{43}-1176 z^{42}- \\
64704 z^{41}+11592 z^{40}+13272 z^{39}+13272 z^{38}+45876 z^{37}+7728 z^{36}+7728 z^{35}+ \\
7728 z^{34}-89412 z^{33}+25032 z^{32}+25704 z^{31}+25704 z^{30}+24192 z^{29}+24192 z^{28}+ \\
22680 z^{27}+22680 z^{26}-109080 z^{25}+43512 z^{24}+42168 z^{23}+42168 z^{22}+5532 z^{21}+ \\
43680 z^{20}+39648 z^{19}+39648 z^{18}+172020 z^{17}+32144 z^{16}+10416 z^{15}+10416 z^{14}- \\
22692 z^{13}+15456 z^{12}+14952 z^{11}+14952 z^{10}-18660 z^{9}+19488 z^{8}+18480 z^{7}+ \\
18480 z^{6}-15636 z^{5}+22512 z^{4}+21000 z^{3}+21000 z^{2}+116628 z+2184\end{array}$ \\
\hline 49 & 149 & $\begin{array}{l}17547 z^{50}+19199 z^{49}+33722 z^{48}+32350 z^{47}+37321 z^{46}+39113 z^{45}+42950 z^{44}+ \\
43608 z^{43}+98001 z^{42}+98351 z^{41}+143378 z^{40}+134362 z^{39}+190369 z^{38}+179617 z^{37}+ \\
226090 z^{36}+205804 z^{35}+279265 z^{34}+254177 z^{33}+314912 z^{32}+277098 z^{31}+ \\
305202 z^{30}+269726 z^{29}+292874 z^{28}+252442 z^{27}+306568 z^{26}+265324 z^{25}+ \\
310126 z^{24}+259558 z^{23}+266266 z^{22}+222110 z^{21}+227222 z^{20}+181470 z^{19}+ \\
195921 z^{18}+156329 z^{17}+167882 z^{16}+125392 z^{15}+88177 z^{14}+60499 z^{13}+29290 z^{12}+ \\
7618 z^{11}+16837 z^{10}+3803 z^{9}+11636 z^{8}-2784 z^{7}-1788 z^{6}-7150 z^{5}-6238 z^{4}- \\
11684 z^{3}-1502 z^{2}-4036 z+4340\end{array}$ \\
\hline & & $228888 z^{149}-195624 z^{150}$ \\
\hline & & $\begin{array}{l}-120 z^{50}-120 z^{48}-36 z^{46}-36 z^{44}-120 z^{42}-120 z^{40}-48 z^{34}-48 z^{32}+108 z^{30}+ \\
108 z^{28}+96 z^{26}+96 z^{24}+288 z^{22}+288 z^{20}+932 z^{18}-296 z^{16}+36 z^{14}+36 z^{12}+ \\
72 z^{10}+72 z^{8}+108 z^{6}+108 z^{4}+156 z^{2}+156\end{array}$ \\
\hline
\end{tabular}




\begin{tabular}{l|l|l} 
& & $179 z^{51}+179 z^{50}+230 z^{49}+230 z^{48}+433 z^{47}+433 z^{46}+481 z^{45}+481 z^{44}+866 z^{43}+$ \\
& & $866 z^{42}+974 z^{41}+974 z^{40}+1329 z^{39}+1329 z^{38}+1317 z^{37}+1317 z^{36}+1561 z^{35}+$ \\
& & $1561 z^{34}+1447 z^{33}+1447 z^{32}+1544 z^{31}+1544 z^{30}+1238 z^{29}+1238 z^{28}+1230 z^{27}+$ \\
& & $1230 z^{26}+906 z^{25}+906 z^{24}+844 z^{23}+844 z^{22}+430 z^{21}+430 z^{20}+353 z^{19}+353 z^{18}-$ \\
& & $z^{17}-z^{16}-36 z^{15}-36 z^{14}-366 z^{13}-366 z^{12}-290 z^{11}-290 z^{10}-422 z^{9}-422 z^{8}-$ \\
& & $211 z^{7}-211 z^{6}-271 z^{5}-271 z^{4}-149 z^{3}-149 z^{2}-152 z-152$ \\
\cline { 3 - 3 } & & $-96 z^{152}+96 z^{153}$ \\
\hline \multirow{2}{*}{51} & \multirow{2}{*}{160} & $4 z^{19}-4 z^{18}-4 z^{17}+4 z^{16}$ \\
\cline { 3 - 3 } & & $z^{52}+z^{48}+2 z^{44}+2 z^{40}+2 z^{36}+2 z^{32}+2 z^{28}+2 z^{24}+2 z^{20}+2 z^{16}+2 z^{12}+2 z^{8}+z^{4}+1$ \\
\cline { 3 - 3 } & & $8 z^{160}-16 z^{161}$ \\
\hline \multicolumn{2}{c|}{$(49,50,51)$} & $-30517344 z^{150}+35706528 z^{149}$ \\
\hline
\end{tabular}

\section{Approximation polynomials and related determinants in Theorem 2}

\begin{tabular}{|c|c|c|}
\hline \multirow{3}{*}{29} & \multirow{3}{*}{89} & $\begin{array}{l}8838 z^{29}-103526 z^{28}+48074 z^{27}+249006 z^{26}-36267 z^{25}-222096 z^{24}-98200 z^{23}- \\
42694 z^{22}+236901 z^{21}+813160 z^{20}+82188 z^{19}+105172 z^{18}-76623 z^{17}-341910 z^{16}- \\
29528 z^{15}+101076 z^{14}+42482 z^{13}+214028 z^{12}-7828 z^{11}-293424 z^{10}-61460 z^{9}+ \\
84522 z^{8}+90968 z^{7}-67100 z^{6}-221774 z^{5}-749016 z^{4}-82002 z^{3}+59754 z^{2}+ \\
157247 z+372960\end{array}$ \\
\hline & & $\begin{array}{l}249196 z^{30}+197392 z^{29}+504078 z^{28}-11390 z^{27}+8214 z^{26}-17793 z^{25}-222796 z^{24}- \\
40936 z^{23}-71854 z^{22}-31047 z^{21}+355216 z^{20}+158208 z^{19}-251360 z^{18}-245645 z^{17}- \\
403198 z^{16}-44440 z^{15}-75264 z^{14}+35828 z^{13}-391672 z^{12}-126276 z^{11}+392512 z^{10}+ \\
300274 z^{9}+635406 z^{8}+79336 z^{7}+155388 z^{6}-30926 z^{5}+185780 z^{4}+8222 z^{3}- \\
417854 z^{2}-180793 z-348900\end{array}$ \\
\hline & & $-341396 z^{89}-904548 z^{90}$ \\
\hline \multirow{3}{*}{30} & \multirow{3}{*}{92} & $\begin{array}{l}284151 z^{30}-303211 z^{29}+34473 z^{28}+230361 z^{27}-272325 z^{26}+122016 z^{25}+ \\
180112 z^{24}-334273 z^{23}+109371 z^{22}+36208 z^{21}-196837 z^{20}+184636 z^{19}+92877 z^{18}- \\
222239 z^{17}+170339 z^{16}+15686 z^{15}-212872 z^{14}+178126 z^{13}+20616 z^{12}-143468 z^{11}+ \\
163829 z^{10}-56575 z^{9}-134101 z^{8}+230702 z^{7}-66928 z^{6}+9672 z^{5}+97210 z^{4}- \\
157573 z^{3}-40095 z^{2}+125240 z-109296\end{array}$ \\
\hline & & $\begin{array}{l}-59086 z^{31}+15283 z^{30}-74369 z^{29}+60109 z^{28}+28737 z^{27}-15235 z^{26}+39866 z^{25}- \\
14610 z^{24}-15469 z^{23}+16375 z^{22}-86676 z^{21}+36259 z^{20}+116870 z^{19}-82905 z^{18}+ \\
67053 z^{17}+31655 z^{16}-103912 z^{15}+26480 z^{14}+172732 z^{13}-187648 z^{12}-74152 z^{11}+ \\
153851 z^{10}-249333 z^{9}+87897 z^{8}+154690 z^{7}-163750 z^{6}-19530 z^{5}+191628 z^{4}- \\
94209 z^{3}-18717 z^{2}+204414 z-206502\end{array}$ \\
\hline & & $379224 z^{92}+172268 z^{93}$ \\
\hline \multirow{3}{*}{31} & \multirow{3}{*}{95} & $\begin{array}{l}-4225266 z^{31}+1316319 z^{30}+2983440 z^{29}-3968815 z^{28}+1611693 z^{27}+1846233 z^{26}- \\
4256835 z^{25}+2557108 z^{24}+1911015 z^{23}-3531513 z^{22}+3405999 z^{21}+155295 z^{20}- \\
3334914 z^{19}+2859543 z^{18}-181131 z^{17}-2388117 z^{16}+2999370 z^{15}-581472 z^{14}- \\
2191518 z^{13}+2452914 z^{12}-917898 z^{11}-1244721 z^{10}+2592741 z^{9}-1622013 z^{8}- \\
1446126 z^{7}+2251806 z^{6}-2410077 z^{5}+236958 z^{4}+2263671 z^{3}-2142621 z^{2}+ \\
587307 z+1506461\end{array}$ \\
\hline & & $\begin{array}{l}303774 z^{32}+398004 z^{31}-205521 z^{30}+755412 z^{29}-338283 z^{28}-77559 z^{27}+87615 z^{26}- \\
492033 z^{25}+285082 z^{24}-79797 z^{23}+281151 z^{22}+665469 z^{21}-1280281 z^{20}-3960 z^{19}+ \\
863013 z^{18}-1755093 z^{17}+770027 z^{16}+705864 z^{15}-1921572 z^{14}+387582 z^{13}+ \\
1832330 z^{12}-1503042 z^{11}+429153 z^{10}+2481123 z^{9}-2516947 z^{8}+797970 z^{7}+ \\
1745100 z^{6}-2355387 z^{5}+325532 z^{4}+1275255 z^{3}-1951479 z^{2}+45261 z+1580655\end{array}$ \\
\hline & & $5421516 z^{95}-3588972 z^{96}$ \\
\hline \multicolumn{2}{|c|}{$(29,30,31)$} & $-127293061031881800 z^{90}-47224605589276632 z^{89}$ \\
\hline
\end{tabular}




\begin{tabular}{|c|c|c|}
\hline \multirow{3}{*}{32} & \multirow{3}{*}{98} & $\begin{array}{l}-22588299 z^{32}+13357080 z^{31}-660150 z^{30}-11245880 z^{29}+13273201 z^{28}- \\
4213080 z^{27}-10169442 z^{26}+14802080 z^{25}-4524244 z^{24}-4588680 z^{23}+ \\
13870419 z^{22}-8577000 z^{21}-3890070 z^{20}+10892280 z^{19}-8706669 z^{18}-1315560 z^{17}+ \\
10323339 z^{16}-8303040 z^{15}-616950 z^{14}+7345200 z^{13}-8432709 z^{12}+1957560 z^{11}+ \\
6776259 z^{10}-9789480 z^{9}+2206179 z^{8}+2264400 z^{7}-9081531 z^{6}+6965040 z^{5}+ \\
2002044 z^{4}-6780360 z^{3}+6752958 z^{2}+61720 z+4831822\end{array}$ \\
\hline & & $\begin{array}{l}1760400 z^{33}+449991 z^{32}+1533720 z^{31}+922782 z^{30}-1734360 z^{29}+1227135 z^{28}- \\
974760 z^{27}-823668 z^{26}+1069520 z^{25}-1197466 z^{24}+2592120 z^{23}+473565 z^{22}- \\
4485800 z^{21}+2729386 z^{20}-1450200 z^{19}-4290933 z^{18}+5043160 z^{17}-1619519 z^{16}- \\
6351840 z^{15}+5718510 z^{14}+2455600 z^{13}-5795075 z^{12}+8621640 z^{11}+1149129 z^{10}- \\
7986200 z^{9}+8595727 z^{8}+484800 z^{7}-7667985 z^{6}+5206240 z^{5}-338900 z^{4}- \\
7833240 z^{3}+6179112 z^{2}+663720 z+4080948\end{array}$ \\
\hline & & $-37889148 z^{98}+9889572 z^{99}$ \\
\hline \multirow{3}{*}{33} & \multirow{3}{*}{101} & $\begin{array}{l}-176064633 z^{33}-85665591 z^{32}+74750757 z^{31}-47272970 z^{30}+21499188 z^{29}+ \\
14694666 z^{28}-95780913 z^{27}+43755587 z^{26}+68776500 z^{25}-4037217 z^{24}+ \\
64432656 z^{23}+27953142 z^{22}-75272931 z^{21}+19707900 z^{20}+4403736 z^{19}- \\
33320676 z^{18}+62965368 z^{17}+7176237 z^{16}-53028738 z^{15}+18240612 z^{14}- \\
16373169 z^{13}-11076483 z^{12}+61498080 z^{11}-43239204 z^{10}-55583784 z^{9}- \\
12887979 z^{8}-46376811 z^{7}-4446624 z^{6}+58106862 z^{5}-9556881 z^{4}+3266673 z^{3}+ \\
35390365 z^{2}+47644845 z+47235177\end{array}$ \\
\hline & & $\begin{array}{l}29638536 z^{34}+24799239 z^{33}+29661303 z^{32}+9226737 z^{31}+15614334 z^{30}+ \\
1923930 z^{29}-24820692 z^{28}-11807025 z^{27}-5265061 z^{26}-5139888 z^{25}+ \\
43840173 z^{24}+25801494 z^{23}-53349362 z^{22}-20883027 z^{21}-5941698 z^{20}- \\
35963706 z^{19}-3232646 z^{18}+17530458 z^{17}-72115791 z^{16}-3288378 z^{15}+ \\
68504836 z^{14}-9686523 z^{13}+50244111 z^{12}+55708260 z^{11}-11129384 z^{10}+ \\
14958006 z^{9}+44808585 z^{8}-49038303 z^{7}-28482668 z^{6}+19260636 z^{5}-58513881 z^{4}+ \\
205749 z^{3}+9770433 z^{2}+39546873 z+55820127\end{array}$ \\
\hline & & $-332338020 z^{101}-144335068 z^{102}$ \\
\hline \multicolumn{2}{|c|}{$(31,32,33)$} & $-445634331745438289520 z^{96}+417178155931626734568 z^{95}$ \\
\hline \multirow{4}{*}{34} & \multirow{3}{*}{104} & $\begin{array}{l}-399703431 z^{34}+1087916661 z^{33}-1630720744 z^{32}-123158126 z^{31}-21796076 z^{30}+ \\
189852624 z^{29}-422979590 z^{28}+46370805 z^{27}+286887150 z^{26}-644304791 z^{25}+ \\
995887336 z^{24}+26439608 z^{23}-84276316 z^{22}+67661308 z^{21}+23651188 z^{20}- \\
47593026 z^{19}+55252839 z^{18}-61203883 z^{17}-32396137 z^{16}+103424620 z^{15}- \\
63992303 z^{14}+4287153 z^{13}+91016151 z^{12}-54334722 z^{11}-247162617 z^{10}+ \\
629737225 z^{9}-1008730988 z^{8}-52474214 z^{7}+164818441 z^{6}-223558699 z^{5}+ \\
266169085 z^{4}+73506901 z^{3}+179099632 z^{2}-630460649 z+1041984688\end{array}$ \\
\hline & & $\begin{array}{l}-94512652 z^{35}+266646659 z^{34}-490549293 z^{33}+857095194 z^{32}+108641546 z^{31}- \\
38038978 z^{30}-12932166 z^{29}-194804864 z^{28}+54188017 z^{27}-85203872 z^{26}- \\
50724817 z^{25}+364466056 z^{24}-113721868 z^{23}-151523710 z^{22}+505358802 z^{21}- \\
756027622 z^{20}+2616640 z^{19}+24294241 z^{18}+176360093 z^{17}-629232539 z^{16}+ \\
29058172 z^{15}+224824483 z^{14}-663863213 z^{13}+1293732477 z^{12}-45638242 z^{11}+ \\
33431723 z^{10}-139780043 z^{9}+284577000 z^{8}+108864730 z^{7}-175863023 z^{6}+ \\
375097749 z^{5}-839815275 z^{4}-44561283 z^{3}+172085310 z^{2}-459202523 z+910810930\end{array}$ \\
\hline & & $-1702388928 z^{104}-837017980 z^{105}$ \\
\hline & & $\begin{array}{l}-1046716196 z^{35}-1705139966 z^{34}+1328534848 z^{33}+209186103 z^{32}- \\
579850958 z^{31}+218235941 z^{30}-105588449 z^{29}+39130547 z^{28}+948112085 z^{27}+ \\
821966732 z^{26}-715563620 z^{25}-86195686 z^{24}-108468120 z^{23}-221335144 z^{22}+ \\
349140044 z^{21}-205764255 z^{20}-57327118 z^{19}+296572370 z^{18}-344630703 z^{17}+ \\
92536691 z^{16}+271430792 z^{15}-441926838 z^{14}+256544717 z^{13}+73948784 z^{12}- \\
877888846 z^{11}-762881152 z^{10}+620094166 z^{9}+185407898 z^{8}+208876518 z^{7}+ \\
575933089 z^{6}-448129406 z^{5}-97527619 z^{4}+781030829 z^{3}+678076638 z^{2}- \\
536082374 z-98863393\end{array}$ \\
\hline
\end{tabular}




\begin{tabular}{|c|c|c|}
\hline & & $\begin{array}{l}144914334 z^{36}+297095560 z^{35}+1169289678 z^{34}-426372626 z^{33}-863497319 z^{32}+ \\
361540034 z^{31}-434779223 z^{30}-368826463 z^{29}+890265147 z^{28}+6887337 z^{27}- \\
155113820 z^{26}+542917024 z^{25}-518948038 z^{24}-879518516 z^{23}-316730730 z^{22}+ \\
557695048 z^{21}-296928951 z^{20}+74727036 z^{19}-279021182 z^{18}-506772977 z^{17}+ \\
1040291399 z^{16}+712099152 z^{15}+918083242 z^{14}-214816021 z^{13}-804575800 z^{12}- \\
106173246 z^{11}+406912124 z^{10}-44306314 z^{9}-497884300 z^{8}+1563142 z^{7}- \\
1043442955 z^{6}-18436418 z^{5}+1246202671 z^{4}+261494749 z^{3}+905060170 z^{2}- \\
152646960 z-822091223\end{array}$ \\
\hline & & $-550045468 z^{107}-1597702416 z^{108}$ \\
\hline & & $\begin{array}{l}-72679207 z^{36}-60042465 z^{35}-27328323 z^{34}+28175850 z^{33}+38242280 z^{32}- \\
1018170 z^{31}-19881498 z^{30}-75330 z^{29}+64690622 z^{28}+35278740 z^{27}+28066812 z^{26}- \\
14776020 z^{25}-50238100 z^{24}-8127540 z^{23}+8674680 z^{22}-1006830 z^{21}+551847 z^{20}+ \\
7718085 z^{19}-12637842 z^{18}-1883655 z^{17}+10669710 z^{16}-9245745 z^{15}+6795732 z^{14}+ \\
6166935 z^{13}-45339792 z^{12}-32359095 z^{11}-28120197 z^{10}+15981300 z^{9}+ \\
56558841 z^{8}+19362645 z^{7}+6299418 z^{6}-10006335 z^{5}+27751201 z^{4}+27999270 z^{3}+ \\
23655654 z^{2}-12371130 z-34995848\end{array}$ \\
\hline 36 & 110 & $\begin{array}{l}3546180 z^{37}+24463035 z^{36}+36719325 z^{35}+40784937 z^{34}-33465960 z^{33}- \\
43519224 z^{32}-7654500 z^{31}-35415090 z^{30}+22383540 z^{29}+35479690 z^{28}- \\
5341950 z^{27}+29684478 z^{26}-8292780 z^{25}-67702526 z^{24}-19643310 z^{23}- \\
9206988 z^{22}+257580 z^{21}+18201351 z^{20}-5600745 z^{19}-45297798 z^{18}+24472935 z^{17}+ \\
84518202 z^{16}+34033365 z^{15}+57933060 z^{14}-29645595 z^{13}-68674026 z^{12}+ \\
9503325 z^{11}+11405619 z^{10}-15313860 z^{9}-26194131 z^{8}-28855035 z^{7}-53742390 z^{6}+ \\
38598525 z^{5}+88270743 z^{4}+28094040 z^{3}+43862046 z^{2}-28627020 z-56682498\end{array}$ \\
\hline & & $-31540680 z^{110}-98505000 z^{111}$ \\
\hline$(34$ & $5,36)$ & $-10749530318906122061682776 z^{105}+39437465990135596375969920 z^{104}$ \\
\hline & & $\begin{array}{l}-328392625 z^{38}+290993025 z^{37}-106716958 z^{36}-80099400 z^{35}+146334448 z^{34}- \\
82673700 z^{33}-10372071 z^{32}+109928850 z^{31}+160680958 z^{30}-236200200 z^{29}+ \\
178868184 z^{28}-25575900 z^{27}-133420172 z^{26}+154610400 z^{25}-68735318 z^{24}- \\
68040750 z^{23}+90264865 z^{22}-26269275 z^{21}-64890392 z^{20}+97028775 z^{19}- \\
58008848 z^{18}-40843875 z^{17}+125617990 z^{16}-79415775 z^{15}-137346362 z^{14}+ \\
191098125 z^{13}-157753650 z^{12}+30459150 z^{11}+147917207 z^{10}-161086425 z^{9}+ \\
76506261 z^{8}+42485775 z^{7}+119836337 z^{6}-146144100 z^{5}+121742572 z^{4}- \\
26396550 z^{3}-74539424 z^{2}+109114050 z-112978051\end{array}$ \\
\hline 38 & 116 & $\begin{array}{l}-9403500 z^{39}+99652845 z^{38}-58716225 z^{37}+37194802 z^{36}-44106150 z^{35}+ \\
13053908 z^{34}+9717150 z^{33}-87002903 z^{32}+120547500 z^{31}-88922450 z^{30}- \\
15160650 z^{29}+146537548 z^{28}-160122600 z^{27}-75053458 z^{26}+189482250 z^{25}- \\
103836420 z^{24}-938400 z^{23}+87715453 z^{22}-87401325 z^{21}-85839656 z^{20}+ \\
176370225 z^{19}+52126768 z^{18}-167935725 z^{17}+205536492 z^{16}-158374125 z^{15}+ \\
13195820 z^{14}+116660925 z^{13}-71477426 z^{12}+1417950 z^{11}-43444389 z^{10}+ \\
16305675 z^{9}-84638221 z^{8}+124726875 z^{7}+67343275 z^{6}-135781050 z^{5}+ \\
126170316 z^{4}-97316700 z^{3}-8126386 z^{2}+76755450 z-33574889\end{array}$ \\
\hline & & $-130668712 z^{116}+529192388 z^{117}$ \\
\hline & & $\begin{array}{l}-149119706349 z^{39}+63707949546 z^{38}-26395317696 z^{37}-36247180542 z^{36}+ \\
59443318176 z^{35}-31786065503 z^{34}-9258370371 z^{33}+37607627380 z^{32}+ \\
80661854706 z^{31}-57012122504 z^{30}+62282372040 z^{29}-7098995408 z^{28}- \\
61235049540 z^{27}+50983686882 z^{26}-20606379006 z^{25}-28591419162 z^{24}+ \\
35781687273 z^{23}-9322212819 z^{22}-30043911930 z^{21}+39553662819 z^{20}- \\
20042522118 z^{19}-18860076597 z^{18}+53019807420 z^{17}-38017902921 z^{16}- \\
67954416972 z^{15}+44264940214 z^{14}-55991529852 z^{13}+15502477726 z^{12}+ \\
68947673487 z^{11}-41919255682 z^{10}+22755960363 z^{9}+17094442241 z^{8}+ \\
57342356871 z^{7}-33076245296 z^{6}+43263248676 z^{5}-12548417012 z^{4}- \\
34528437732 z^{3}+38395278251 z^{2}-43071249867 z+10796816534\end{array}$ \\
\hline
\end{tabular}




\begin{tabular}{|c|c|c|}
\hline & & $\begin{array}{l}3019569924 z^{40}+46348505073 z^{39}+12526842752 z^{38}+10272887556 z^{37}- \\
25924554292 z^{36}+1310254080 z^{35}-18755197493 z^{34}-35923872231 z^{33}+ \\
58445245834 z^{32}-31003013634 z^{31}+5501615364 z^{30}+62566901664 z^{29}- \\
71660413140 z^{28}-45616511226 z^{27}+49880906186 z^{26}-32766551160 z^{25}- \\
23248226440 z^{24}+38184226665 z^{23}-46360350849 z^{22}-41824933494 z^{21}+ \\
97154733705 z^{20}+38120227062 z^{19}-17090569745 z^{18}+76837413774 z^{17}- \\
60618822959 z^{16}-4884457938 z^{15}+41319863530 z^{14}-24244285020 z^{13}- \\
24745519454 z^{12}-21728806557 z^{11}-28922373896 z^{10}-33575376855 z^{9}+ \\
69559600537 z^{8}+41361916065 z^{7}-9475846394 z^{6}+44214361152 z^{5}- \\
34357388522 z^{4}-10977837426 z^{3}+18941010169 z^{2}-9408704265 z-13383501602 \\
-484474868376 z^{119}-461974967404 z^{120}\end{array}$ \\
\hline \multirow{3}{*}{40} & \multirow{3}{*}{122} & $\begin{array}{l}91864726291 z^{40}+319280781460 z^{39}-224030950487 z^{38}+27374191880 z^{37}+ \\
292533708140 z^{36}-292674605820 z^{35}-1510481652 z^{34}+44669145100 z^{33}- \\
334085225207 z^{32}-134799607080 z^{31}+317018563878 z^{30}-254112973440 z^{29}- \\
145256148136 z^{28}+252753057880 z^{27}-182489746860 z^{26}+36049279040 z^{25}+ \\
258332973123 z^{24}-158528733880 z^{23}-48928115708 z^{22}+193350332700 z^{21}- \\
140746785575 z^{20}+38800707260 z^{19}+159559557623 z^{18}-333737842620 z^{17}+ \\
34029855687 z^{16}+103226012960 z^{15}-240864919868 z^{14}+290346874040 z^{13}+ \\
113028048961 z^{12}-180497459260 z^{11}+172178300702 z^{10}-72252586560 z^{9}- \\
256768685827 z^{8}-94832110460 z^{7}+161086577681 z^{6}-220632804400 z^{5}- \\
82608922550 z^{4}+163568737100 z^{3}-139940524770 z^{2}+286924169860 z+ \\
198899712593\end{array}$ \\
\hline & & $\begin{array}{l}58079840240 z^{41}+20779678345 z^{40}+103515938500 z^{39}-17011134207 z^{38}- \\
147808517040 z^{37}+59923127422 z^{36}-212289811620 z^{35}-13631116930 z^{34}+ \\
321426076900 z^{33}-153779160721 z^{32}+233225695720 z^{31}+128766125602 z^{30}- \\
434954929680 z^{29}+207808787358 z^{28}+64712750720 z^{27}-349503491512 z^{26}- \\
73802320960 z^{25}+81918338683 z^{24}-322745890720 z^{23}+126471217758 z^{22}+ \\
533202716180 z^{21}-303556927977 z^{20}+206915469740 z^{19}+290555338185 z^{18}- \\
398457916780 z^{17}+132114286577 z^{16}+39320376200 z^{15}-306305865594 z^{14}- \\
132039615160 z^{13}+68052119533 z^{12}-229173884420 z^{11}+57726516830 z^{10}+ \\
402305026120 z^{9}-200625842251 z^{8}+162582080740 z^{7}+207397909869 z^{6}- \\
204794211720 z^{5}+97428157174 z^{4}-17980049220 z^{3}-177803934800 z^{2}- \\
84951726820 z+25430355927\end{array}$ \\
\hline & & $-537183155528 z^{122}-703138211928 z^{123}$ \\
\hline \multicolumn{2}{|c|}{$(38,39,40)$} & $1070663185232794219680724801040 z^{117}-383714096776182783428173226848 z^{116}$ \\
\hline & & $\begin{array}{l}654719760512 z^{43}-2821346833612 z^{42}-94694672644 z^{41}+2479488890145 z^{40}- \\
512912959904 z^{39}+2552059180057 z^{38}+90318251584 z^{37}-540036131793 z^{36}- \\
332932250408 z^{35}+689404484714 z^{34}-233019909196 z^{33}-1816808882738 z^{32}+ \\
350569737040 z^{31}-298843735826 z^{30}+80388796672 z^{29}-759331507046 z^{28}- \\
193863522676 z^{27}+98917002890 z^{26}+239024931320 z^{25}+507105169032 z^{24}+ \\
55088360924 z^{23}+45426928350 z^{22}-450655798776 z^{21}-92389707567 z^{20}+ \\
127506668620 z^{19}-104589492891 z^{18}+423189766836 z^{17}-553853895119 z^{16}- \\
211431381744 z^{15}-129812189536 z^{14}-130698692896 z^{13}+643148743452 z^{12}- \\
340716989224 z^{11}+2775000173564 z^{10}-160997824948 z^{9}-2755147607899 z^{8}+ \\
301462445928 z^{7}-2157483293747 z^{6}+366504279088 z^{5}+596005489469 z^{4}+ \\
138760078784 z^{3}-725373895320 z^{2}-128931956988 z+2197279449742\end{array}$ \\
\hline
\end{tabular}




\begin{tabular}{|c|c|c|}
\hline & & $\begin{array}{l}1600354283224 z^{44}+229103252712 z^{43}-1515378673264 z^{42}-223609710724 z^{41}+ \\
626899969097 z^{40}-411335257848 z^{39}+1782268460381 z^{38}+514927759856 z^{37}- \\
1635050608811 z^{36}+471980486624 z^{35}-2021350873784 z^{34}-698337174500 z^{33}+ \\
2025446830184 z^{32}-67928635024 z^{31}+2924131042610 z^{30}-13100256640 z^{29}- \\
1559320311274 z^{28}-450365666348 z^{27}-1826792092706 z^{26}+681361714048 z^{25}+ \\
1581036201434 z^{24}+469409501316 z^{23}-2128757496492 z^{22}-625900977864 z^{21}+ \\
1072970184197 z^{20}-161130674252 z^{19}+4404057533735 z^{18}+2130376028 z^{17}- \\
3832527512063 z^{16}-342823093552 z^{15}-1230015018030 z^{14}+496838785344 z^{13}+ \\
1676061259314 z^{12}+420162704880 z^{11}-2827316268964 z^{10}-350037673132 z^{9}+ \\
1078126371345 z^{8}-203066232000 z^{7}+4111288791141 z^{6}-26920017216 z^{5}- \\
2150284069005 z^{4}+30602791784 z^{3}-1803737859838 z^{2}-50999731780 z+ \\
1712645539000\end{array}$ \\
\hline & & $1922418947808 z^{131}+6043431728416 z^{132}$ \\
\hline \multirow{4}{*}{44} & \multirow{3}{*}{134} & $\begin{array}{l}-75947033832 z^{44}-5579107092 z^{43}-104238012156 z^{42}+4739827911 z^{41}+ \\
105950111578 z^{40}+4937188815 z^{39}+95161460838 z^{38}-1864833711 z^{37}+ \\
82640354966 z^{36}+1664230542 z^{35}+19169079328 z^{34}-1874055534 z^{33}- \\
110419687504 z^{32}-2242997406 z^{31}+2455649268 z^{30}-41909634 z^{29}- \\
71433888516 z^{28}+614444454 z^{27}+4379526516 z^{26}-773138496 z^{25}+ \\
38156981404 z^{24}+947504922 z^{23}-11826042596 z^{22}+1292556447 z^{21}- \\
355903374 z^{20}-2056683525 z^{19}+16466899058 z^{18}-1263948465 z^{17}- \\
53825333390 z^{16}+1616137536 z^{15}-20478872416 z^{14}+74222772 z^{13}+ \\
120456198304 z^{12}+4671106596 z^{11}+104403966276 z^{10}-3272846733 z^{9}- \\
123793016934 z^{8}-5059153749 z^{7}-66892041266 z^{6}+393363555 z^{5}-64852742270 z^{4}+ \\
298156560 z^{3}-41110831292 z^{2}+2510715714 z+140992632208\end{array}$ \\
\hline & & $\begin{array}{l}2216770296 z^{45}+57129877336 z^{44}-2728300848 z^{43}-60713752852 z^{42}+ \\
2166681231 z^{41}+40970060514 z^{40}+2967191643 z^{39}+77208074222 z^{38}- \\
4780052277 z^{37}-97090594078 z^{36}-2387742864 z^{35}-96526339780 z^{34}+ \\
5426834712 z^{33}+90857543716 z^{32}+2175781374 z^{31}+132551744076 z^{30}- \\
167355630 z^{29}-46431083436 z^{28}-2857116366 z^{27}-61093058028 z^{26}- \\
1027769106 z^{25}+6007230904 z^{24}-935469756 z^{23}-111414475152 z^{22}+ \\
2497628523 z^{21}+96742259546 z^{20}+5218568745 z^{19}+185829175462 z^{18}- \\
3930963153 z^{17}-131339449078 z^{16}-2434706274 z^{15}-36798869300 z^{14}+ \\
377423982 z^{13}+10056749292 z^{12}-2820430860 z^{11}-128752306132 z^{10}+ \\
1823523351 z^{9}+71211869794 z^{8}+6026206563 z^{7}+164593057134 z^{6}-2461736979 z^{5}- \\
56421410130 z^{4}-3939916266 z^{3}-63767607912 z^{2}+3454515480 z+25963043060\end{array}$ \\
\hline & & $-512186985504 z^{134}-522891150768 z^{135}$ \\
\hline & & $\begin{array}{l}541293377520 z^{45}-968893495884 z^{44}-1198079333952 z^{43}+447173795391 z^{42}+ \\
777341459536 z^{41}-88091856171 z^{40}+1040857866600 z^{39}+15100602735 z^{38}- \\
1111190897800 z^{37}+1538190196584 z^{36}+371502990856 z^{35}-964937789790 z^{34}+ \\
168304420664 z^{33}-170359771302 z^{32}-770970946800 z^{31}+147538176606 z^{30}+ \\
383820068304 z^{29}-1091557612278 z^{28}+246926658864 z^{27}+587156627544 z^{26}- \\
516091336808 z^{25}+390068663154 z^{24}+288431248168 z^{23}-1057237233597 z^{22}+ \\
510827218944 z^{21}+346537536069 z^{20}-713212429168 z^{19}+9374023066655 z^{18}- \\
174655591352 z^{17}-1070279230902 z^{16}+671183463200 z^{15}-291468004068 z^{14}- \\
735491160272 z^{13}+1861353055764 z^{12}+812032969152 z^{11}-981007879581 z^{10}- \\
176504582496 z^{9}-240996892647 z^{8}-1161587791976 z^{7}+1040368757721 z^{6}+ \\
435701866840 z^{5}-1674922778490 z^{4}+365855876968 z^{3}+103783298334 z^{2}- \\
50206939352 z+1047984167376\end{array}$ \\
\hline
\end{tabular}




\begin{tabular}{|c|c|c|}
\hline & & $\begin{array}{l}375826069824 z^{46}+389037204976 z^{45}-51783796104 z^{44}-564345721792 z^{43}- \\
316470592089 z^{42}+407337506496 z^{41}+65331297489 z^{40}+607967063384 z^{39}+ \\
718385445921 z^{38}-941731846600 z^{37}-402580388634 z^{36}-318233580952 z^{35}- \\
1105125087036 z^{34}+1260902030248 z^{33}+928573409322 z^{32}-214019408016 z^{31}- \\
139378083822 z^{30}+240560920848 z^{29}-848300707458 z^{28}-355077245616 z^{27}+ \\
1794280909542 z^{26}-467081765816 z^{25}-922373507592 z^{24}+312051187944 z^{23}- \\
1321527938829 z^{22}+256058214800 z^{21}+1870245875859 z^{20}+455245169152 z^{19}- \\
685952191995 z^{18}-410889238072 z^{17}-463142659512 z^{16}-436655545808 z^{15}+ \\
1478403246906 z^{14}-1591217568 z^{13}-1100965032744 z^{12}-137175061936 z^{11}- \\
672625854345 z^{10}+130115107936 z^{9}+1183740872769 z^{8}+880615121736 z^{7}- \\
470011423005 z^{6}-356422892616 z^{5}+207556342200 z^{4}-913159201752 z^{3}+ \\
369523810776 z^{2}+926067028376 z-751138064892\end{array}$ \\
\hline & & $-4505097105248 z^{137}-544781681936 z^{138}$ \\
\hline (43) & 45) & $\begin{array}{l}-561535751331132078651839819235968256 z^{132} \\
255900474003210216622468298452205568 z^{131}\end{array}$ \\
\hline & & 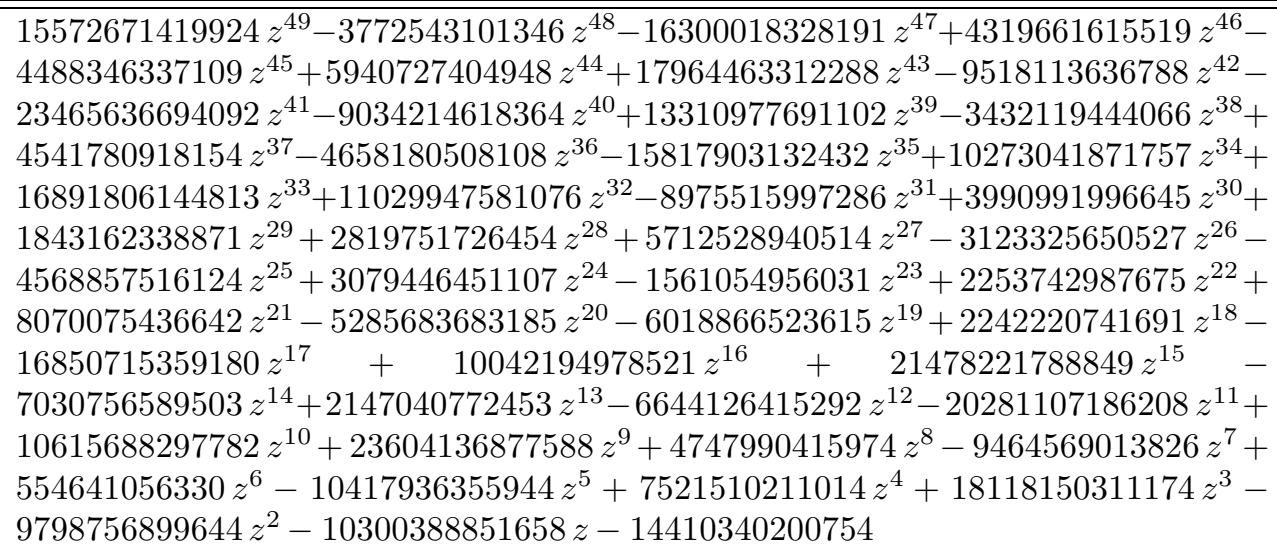 \\
\hline 49 & 149 & $\begin{array}{l}-4599090143590 z^{50}+47240971614 z^{49}-7371501379626 z^{48}+3684666222245 z^{47}- \\
2239828388971 z^{46}-12147728070459 z^{45}+1592748784080 z^{44}+8564296897524 z^{43}- \\
965625646126 z^{42}+10131713521668 z^{41}-11133490379610 z^{40}-15177903407224 z^{39}+ \\
3577268952172 z^{38}-2784273097486 z^{37}+11204317248478 z^{36}+14206959307062 z^{35}- \\
6344191607115 z^{34}-2797061583877 z^{33}-6009296669398 z^{32}-9068120053960 z^{31}+ \\
2840232781361 z^{30}+11928343993591 z^{29}-7364408409198 z^{28}-1772349624642 z^{27}+ \\
496840832203 z^{26}-19016054531576 z^{25}+9858371330193 z^{24}+19295872493353 z^{23}- \\
4521827637741 z^{22}+1208342237300 z^{21}-4374640110055 z^{20}-18502749486377 z^{19}+ \\
6104435716535 z^{18}+19146661474462 z^{17}+488274966683 z^{16}-3316326725319 z^{15}+ \\
2182282334815 z^{14}-14824597559769 z^{13}+7532277927728 z^{12}+16314787492980 z^{11}- \\
6917870993576 z^{10}-723482062748 z^{9}-8442497375404 z^{8}-10800247171176 z^{7}+ \\
1817577563136 z^{6}+7697242742116 z^{5}-521297676820 z^{4}+2233470317936 z^{3}- \\
420000649522 z^{2}-2445166706486 z-554792510556\end{array}$ \\
\hline & & $-44864565312812 z^{149}-68768563833944 z^{150}$ \\
\hline
\end{tabular}




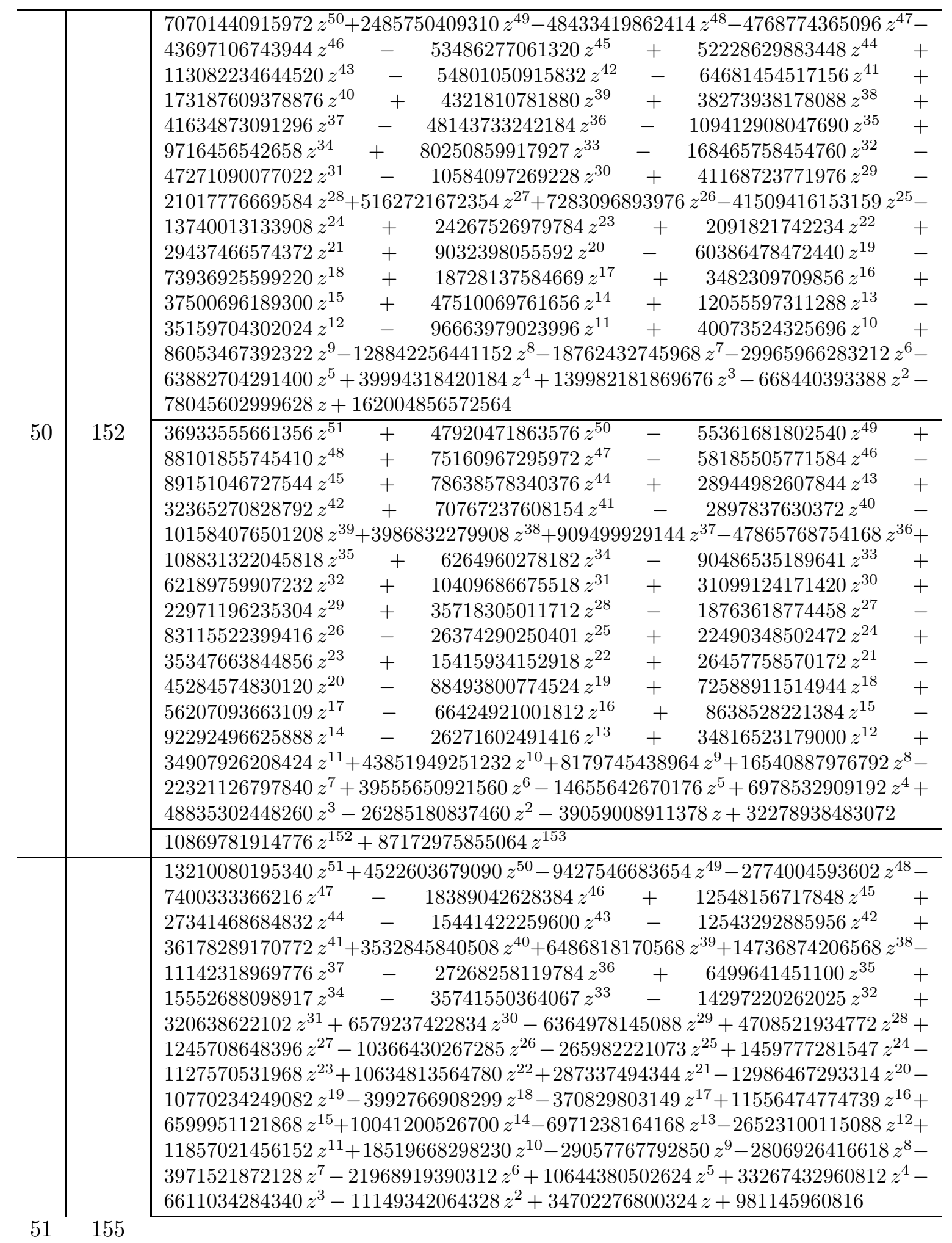




\begin{tabular}{|c|c|}
\hline & 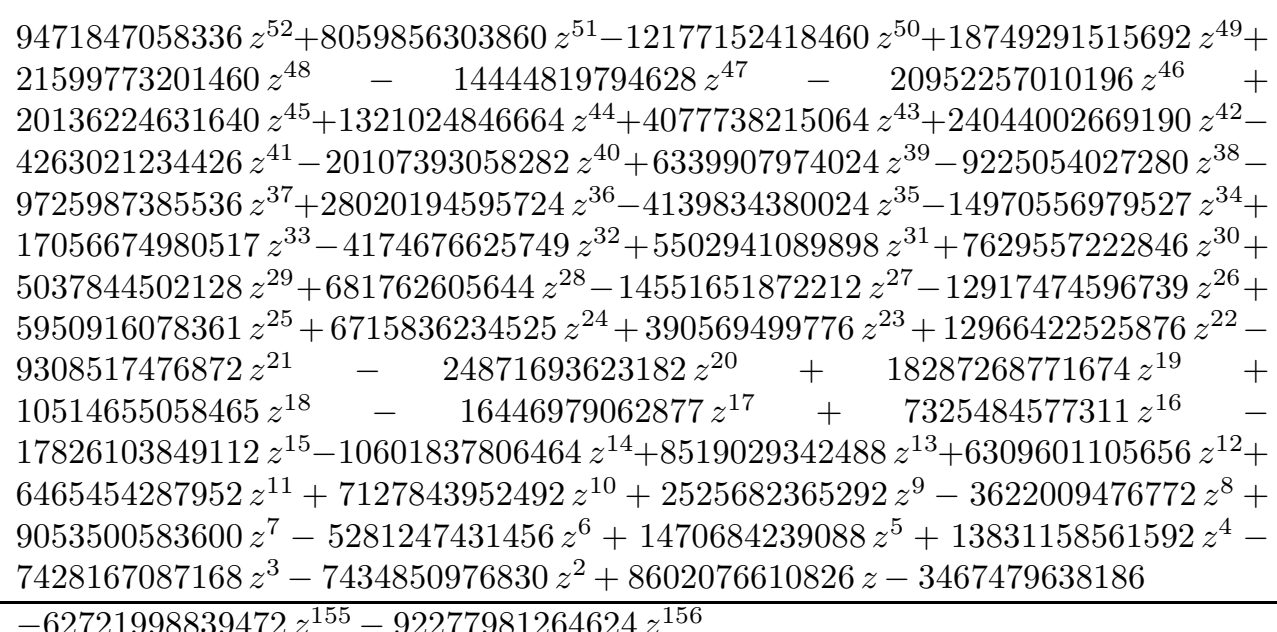 \\
\hline & $\begin{array}{l}-62721998839472 z^{155}-92277981264624 z^{156} \\
14680803386096826096942113490747540708160 z^{150} \\
26623480618461132219167377379353660824672 z^{149}\end{array}$ \\
\hline
\end{tabular}

\section{Approximation polynomials and related determinants in Theorem 3}

\begin{tabular}{|c|c|c|}
\hline \multirow{3}{*}{19} & \multirow{3}{*}{59} & $-153 z^{19}-4 z^{18}+z^{17}+15 z^{13}+z^{11}+56 z^{10}+12 z^{9}+15 z^{7}+z^{5}+56 z-4$ \\
\hline & & $153 z^{19}-4 z^{18}-z^{17}-15 z^{13}-z^{11}+56 z^{10}-12 z^{9}-15 z^{7}-z^{5}-56 z-4$ \\
\hline & & $2 z^{59}+30 z^{61}$ \\
\hline \multirow{3}{*}{20} & \multirow{3}{*}{62} & $41 z^{20}+z^{19}-3 z^{18}-4 z^{14}-15 z^{11}-3 z^{10}+z^{9}-4 z^{8}-15 z^{2}+z+1$ \\
\hline & & $-41 z^{20}+z^{19}+3 z^{18}+4 z^{14}-15 z^{11}+3 z^{10}+z^{9}+4 z^{8}+15 z^{2}+z-1$ \\
\hline & & $-8 z^{62}-8 z^{64}$ \\
\hline \multirow{3}{*}{21} & \multirow{3}{*}{65} & $76 z^{21}+2 z^{20}-6 z^{19}-2 z^{18}-8 z^{15}-28 z^{12}-6 z^{11}+2 z^{10}-2 z^{9}-28 z^{3}+2 z^{2}+2 z-2$ \\
\hline & & $-76 z^{21}+2 z^{20}+6 z^{19}-2 z^{18}+8 z^{15}-28 z^{12}+6 z^{11}+2 z^{10}+2 z^{9}+28 z^{3}+2 z^{2}-2 z-2$ \\
\hline & & $-16 z^{65}-16 z^{69}$ \\
\hline \multicolumn{2}{|c|}{$(19,20,21)$} & $-8 z^{59}$ \\
\hline \multirow{3}{*}{26} & \multirow{3}{*}{80} & $\begin{array}{l}-379 z^{26}+z^{25}+z^{24}+11 z^{23}+z^{22}+z^{21}+11 z^{20}+z^{19}+z^{18}+141 z^{17}+z^{16}+z^{15}+ \\
11 z^{14}+z^{13}+z^{12}+11 z^{11}+z^{10}+z^{9}+141 z^{8}+z^{7}+z^{6}+11 z^{5}+z^{4}+z^{3}+11 z^{2}+z+1\end{array}$ \\
\hline & & $\begin{array}{l}379 z^{26}+z^{25}-z^{24}+11 z^{23}-z^{22}+z^{21}-11 z^{20}+z^{19}-z^{18}+141 z^{17}-z^{16}+z^{15}- \\
11 z^{14}+z^{13}-z^{12}+11 z^{11}-z^{10}+z^{9}-141 z^{8}+z^{7}-z^{6}+11 z^{5}-z^{4}+z^{3}-11 z^{2}+z-1\end{array}$ \\
\hline & & $-1040 z^{80}+2 z^{82}$ \\
\hline \multirow{3}{*}{27} & \multirow{3}{*}{83} & $\begin{array}{l}1798 z^{27}-z^{26}-z^{25}-11 z^{24}-z^{23}-z^{22}-11 z^{21}-z^{20}-z^{19}-141 z^{18}-z^{17}-z^{16}-11 z^{15}- \\
z^{14}-z^{13}-11 z^{12}-z^{11}-z^{10}-141 z^{9}-z^{8}-z^{7}-11 z^{6}-z^{5}-z^{4}-11 z^{3}-z^{2}-z-1419\end{array}$ \\
\hline & & $\begin{array}{l}-z^{26}+z^{25}-11 z^{24}+z^{23}-z^{22}+11 z^{21}-z^{20}+z^{19}-141 z^{18}+z^{17}-z^{16}+11 z^{15}- \\
z^{14}+z^{13}-11 z^{12}+z^{11}-z^{10}+141 z^{9}-z^{8}+z^{7}-11 z^{6}+z^{5}-z^{4}+11 z^{3}-z^{2}+z+379\end{array}$ \\
\hline & & $-2 z^{83}-2 z^{85}$ \\
\hline \multirow{3}{*}{28} & \multirow{3}{*}{108} & $z^{28}+z^{27}-z-1$ \\
\hline & & $z^{28}+z^{27}+z+1$ \\
\hline & & $2 z^{108}+2 z^{109}$ \\
\hline \multicolumn{2}{|c|}{$(26,27,28)$} & $1081600 z^{81}+1081600 z^{80}$ \\
\hline \multirow{3}{*}{39} & \multirow{3}{*}{119} & $z^{39}+z^{38}-z^{12}-z^{11}$ \\
\hline & & $z^{39}+z^{38}+z^{12}+z^{11}$ \\
\hline & & $2 z^{119}+2 z^{120}$ \\
\hline \multirow[b]{2}{*}{40} & \multirow[b]{2}{*}{136} & $\begin{array}{l}-226980 z^{40}+16380 z^{38}+16380 z^{36}+18720 z^{34}+18720 z^{28}+35100 z^{22}-2340 z^{20}- \\
2340 z^{18}+131040 z^{13}-9360 z^{11}-9360 z^{9}-9360 z^{7}+35100 z^{4}-2340 z^{2}-9360 z-2340\end{array}$ \\
\hline & & $\begin{array}{l}226980 z^{40}-16380 z^{38}-16380 z^{36}-18720 z^{34}-18720 z^{28}-35100 z^{22}+2340 z^{20}+ \\
2340 z^{18}+131040 z^{13}-9360 z^{11}-9360 z^{9}-9360 z^{7}-35100 z^{4}+2340 z^{2}-9360 z+2340\end{array}$ \\
\hline
\end{tabular}




\begin{tabular}{l|l|l}
\cline { 3 - 3 } & & $18720 z^{136}+18720 z^{142}$ \\
\hline \multirow{4}{*}{41} & $-2340 z^{41}-226980 z^{39}+17836 z^{37}+1552 z^{35}+18832 z^{33}+1552 z^{29}+18832 z^{27}+$ \\
& \multirow{5}{*}{135} & $556 z^{23}+35116 z^{21}-2548 z^{19}+1448 z^{14}+131048 z^{12}-10192 z^{10}-776 z^{8}-9416 z^{6}+$ \\
\cline { 3 - 3 } & $556 z^{5}+35116 z^{3}-776 z^{2}-2548 z-9416$ \\
\cline { 3 - 3 } & & $2340 z^{41}+226980 z^{39}-17836 z^{37}-1552 z^{35}-18832 z^{33}-1552 z^{29}-18832 z^{27}-$ \\
& $556 z^{23}-35116 z^{21}+2548 z^{19}+1448 z^{14}+131048 z^{12}-10192 z^{10}-776 z^{8}-9416 z^{6}-$ \\
& $556 z^{5}-35116 z^{3}-776 z^{2}+2548 z-9416$ \\
\cline { 3 - 3 } & $18832 z^{135}+1552 z^{137}$ \\
\hline \multicolumn{2}{l|}{$(39,40,41)$} & $88133760 z^{119}(1+z)$ \\
\hline
\end{tabular}

\section{Approximation polynomials and related determinants in Theorem 4}

\begin{tabular}{|c|c|c|}
\hline \multirow{3}{*}{17} & \multirow{3}{*}{53} & $z^{17}+z^{16}-z^{15}+z^{14}-z^{5}-z^{4}-z^{3}+z^{2}$ \\
\hline & & $z^{17}-z^{16}-z^{15}-z^{14}-z^{5}+z^{4}-z^{3}-z^{2}$ \\
\hline & & $16 z^{53}-16 z^{61}$ \\
\hline \multirow{3}{*}{18} & \multirow{3}{*}{56} & $\begin{array}{l}2 z^{18}-6 z^{17}-5 z^{16}-z^{15}+3 z^{14}+z^{13}-z^{12}+3 z^{11}-3 z^{10}-z^{9}-z^{8}+3 z^{7}+3 z^{6}+ \\
z^{5}+z^{4}+5 z^{3}-3 z^{2}-5 z-8\end{array}$ \\
\hline & & $\begin{array}{l}2 z^{18}+6 z^{17}-5 z^{16}+z^{15}+3 z^{14}-z^{13}-z^{12}-3 z^{11}-3 z^{10}+z^{9}-z^{8}-3 z^{7}+3 z^{6}- \\
z^{5}+z^{4}-5 z^{3}-3 z^{2}+5 z-8\end{array}$ \\
\hline & & $-64 z^{56}-64 z^{58}$ \\
\hline \multirow{3}{*}{19} & \multirow{3}{*}{59} & $\begin{array}{l}z^{19}+z^{18}+z^{17}-z^{16}-z^{15}-z^{14}+z^{13}-z^{12}+z^{11}+z^{10}+z^{9}-z^{8}-z^{7}-z^{6}+z^{5}- \\
z^{4}+2 z^{3}+2 z^{2}+2 z-2\end{array}$ \\
\hline & & $\begin{array}{l}z^{19}-z^{18}+z^{17}+z^{16}-z^{15}+z^{14}+z^{13}+z^{12}+z^{11}-z^{10}+z^{9}+z^{8}-z^{7}+z^{6}+z^{5}+ \\
z^{4}+2 z^{3}-2 z^{2}+2 z+2\end{array}$ \\
\hline & & $32 z^{59}+32 z^{67}$ \\
\hline \multicolumn{2}{|c|}{$(17,18,19)$} & $-512 z^{53}$ \\
\hline \multirow{3}{*}{21} & \multirow{3}{*}{65} & $\begin{array}{l}2 z^{21}+2 z^{20}-2 z^{19}+2 z^{18}-z^{17}-z^{16}-z^{15}+z^{14}-z^{13}-z^{12}-3 z^{11}+3 z^{10}-z^{9}- \\
z^{8}-5 z^{7}+5 z^{6}-z^{5}-z^{4}-3 z^{3}+3 z^{2}-4 z-4\end{array}$ \\
\hline & & $\begin{array}{l}2 z^{21}-2 z^{20}-2 z^{19}-2 z^{18}-z^{17}+z^{16}-z^{15}-z^{14}-z^{13}+z^{12}-3 z^{11}-3 z^{10}-z^{9}+ \\
z^{8}-5 z^{7}-5 z^{6}-z^{5}+z^{4}-3 z^{3}-3 z^{2}-4 z+4\end{array}$ \\
\hline & & $-64 z^{65}-64 z^{69}$ \\
\hline \multirow{3}{*}{22} & \multirow{3}{*}{70} & $\begin{array}{l}4 z^{22}-4 z^{21}+z^{20}+z^{19}+z^{18}-z^{17}-2 z^{16}-2 z^{15}+4 z^{14}+4 z^{13}+6 z^{12}-2 z^{11}- \\
4 z^{10}-4 z^{9}+4 z^{8}-4 z^{7}+2 z^{6}+6 z^{5}+3 z^{4}-5 z^{3}+5 z^{2}+3 z-8\end{array}$ \\
\hline & & $\begin{array}{l}4 z^{22}+4 z^{21}+z^{20}-z^{19}+z^{18}+z^{17}-2 z^{16}+2 z^{15}+4 z^{14}-4 z^{13}+6 z^{12}+2 z^{11}- \\
4 z^{10}+4 z^{9}+4 z^{8}+4 z^{7}+2 z^{6}-6 z^{5}+3 z^{4}+5 z^{3}+5 z^{2}-3 z-8\end{array}$ \\
\hline & & $128 z^{70}-64 z^{72}$ \\
\hline \multirow{3}{*}{23} & \multirow{3}{*}{71} & $\begin{array}{l}4 z^{23}-4 z^{22}+z^{21}+z^{20}+z^{19}-z^{18}-2 z^{17}-2 z^{16}+z^{15}+z^{14}+3 z^{13}+z^{12}-z^{11}- \\
z^{10}+z^{9}-z^{8}-z^{7}+3 z^{6}-2 z^{4}+2 z^{3}-5 z-3\end{array}$ \\
\hline & & $\begin{array}{l}4 z^{23}+4 z^{22}+z^{21}-z^{20}+z^{19}+z^{18}-2 z^{17}+2 z^{16}+z^{15}-z^{14}+3 z^{13}-z^{12}-z^{11}+ \\
z^{10}+z^{9}+z^{8}-z^{7}-3 z^{6}+2 z^{4}+2 z^{3}-5 z+3\end{array}$ \\
\hline & & $32 z^{71}-64 z^{73}$ \\
\hline \multicolumn{2}{|c|}{$(21,22,23)$} & $3072 z^{65}$ \\
\hline \multirow{4}{*}{26} & \multirow{3}{*}{80} & $\begin{array}{l}8 z^{26}+5 z^{24}-3 z^{23}+5 z^{22}-5 z^{21}-z^{20}-z^{19}+3 z^{18}+z^{17}+4 z^{16}-4 z^{13}+2 z^{12}- \\
2 z^{11}+2 z^{10}+2 z^{9}+3 z^{8}-z^{7}+3 z^{6}+z^{5}-5 z^{4}-z^{3}-5 z^{2}-3 z-8\end{array}$ \\
\hline & & $\begin{array}{l}8 z^{26}+5 z^{24}+3 z^{23}+5 z^{22}+5 z^{21}-z^{20}+z^{19}+3 z^{18}-z^{17}+4 z^{16}+4 z^{13}+2 z^{12}+ \\
2 z^{11}+2 z^{10}-2 z^{9}+3 z^{8}+z^{7}+3 z^{6}-z^{5}-5 z^{4}+z^{3}-5 z^{2}+3 z-8\end{array}$ \\
\hline & & $64 z^{80}+64 z^{88}$ \\
\hline & & $\begin{array}{l}4 z^{27}+4 z^{26}+4 z^{25}-4 z^{24}+2 z^{19}+2 z^{18}+2 z^{17}-2 z^{16}-2 z^{15}-2 z^{14}+2 z^{13}- \\
2 z^{12}+2 z^{11}+2 z^{10}+2 z^{9}-2 z^{8}+2 z^{7}+2 z^{6}-2 z^{5}+2 z^{4}-4 z^{3}-4 z^{2}-4 z+4 \\
\end{array}$ \\
\hline
\end{tabular}




\begin{tabular}{|c|c|c|}
\hline & & $\begin{array}{l}4 z^{27}-4 z^{26}+4 z^{25}+4 z^{24}+2 z^{19}-2 z^{18}+2 z^{17}+2 z^{16}-2 z^{15}+2 z^{14}+2 z^{13}+ \\
2 z^{12}+2 z^{11}-2 z^{10}+2 z^{9}+2 z^{8}+2 z^{7}-2 z^{6}-2 z^{5}-2 z^{4}-4 z^{3}+4 z^{2}-4 z-4\end{array}$ \\
\hline & & $128 z^{91}+64 z^{99}$ \\
\hline \multirow{3}{*}{28} & \multirow{3}{*}{88} & $\begin{array}{l}2 z^{28}+4 z^{27}+8 z^{26}+4 z^{25}+2 z^{24}-4 z^{23}+4 z^{22}-4 z^{21}+z^{19}+3 z^{18}+3 z^{17}+2 z^{16}- \\
2 z^{15}-2 z^{14}-2 z^{13}-2 z^{11}+4 z^{10}+2 z^{9}+2 z^{8}+4 z^{6}-2 z^{4}-z^{3}-9 z^{2}-3 z-6\end{array}$ \\
\hline & & $\begin{array}{l}2 z^{28}+4 z^{26}+6 z^{24}+4 z^{23}+4 z^{22}+4 z^{21}+z^{19}+z^{18}-z^{17}+4 z^{16}+4 z^{13}+2 z^{12}+ \\
4 z^{11}+2 z^{10}+4 z^{8}+2 z^{7}+2 z^{6}-2 z^{5}-4 z^{4}-3 z^{3}-5 z^{2}-z-10\end{array}$ \\
\hline & & $64 z^{88}+128 z^{90}$ \\
\hline \multicolumn{2}{|c|}{$(26,27,28)$} & $-4096 z^{80}$ \\
\hline
\end{tabular}

\section{Approximation polynomials and related determinants in Theorem 5}

\begin{tabular}{|c|c|c|}
\hline \multirow{3}{*}{10} & \multirow{3}{*}{31} & $z^{10}-z^{9}-z^{8}+z^{6}-z^{5}-2 z^{4}+z^{2}-z$ \\
\hline & & $z^{9}+2 z^{8}-z^{7}+3 z^{5}+2 z^{4}+1$ \\
\hline & & $z^{31}-z^{47}$ \\
\hline \multirow{3}{*}{11} & \multirow{3}{*}{43} & $z^{9}+z^{5}+z^{4}+z+1$ \\
\hline & & $-z^{10}-z^{9}-z^{8}-z^{6}-2 z^{5}-z^{4}-z^{3}-z^{2}-2 z-2$ \\
\hline & & $z^{43}-z^{64}$ \\
\hline \multirow{3}{*}{12} & \multirow{3}{*}{43} & $z^{10}+3 z^{9}+z^{6}+z^{5}+z^{4}+z^{2}+z+1$ \\
\hline & & $-z^{11}-4 z^{10}-4 z^{9}-3 z^{8}-z^{7}-2 z^{6}-2 z^{5}+z^{4}-2 z^{3}-2 z^{2}-3 z-4$ \\
\hline & & $z^{43}-2 z^{44}$ \\
\hline \multicolumn{2}{|c|}{$(10,11,12)$} & $-3 z^{32}-2 z^{31}$ \\
\hline \multirow{3}{*}{26} & \multirow{3}{*}{79} & $z^{24}+z^{20}+z^{16}$ \\
\hline & & $-z^{25}-z^{24}-z^{23}-z^{21}-z^{20}-z^{17}-z^{16}-z^{15}$ \\
\hline & & $-z^{79}-z^{207}$ \\
\hline \multirow{3}{*}{27} & \multirow{3}{*}{83} & $-z^{24}-z^{20}+z^{4}+1$ \\
\hline & & $z^{25}+z^{24}+z^{21}+z^{20}+z^{19}-z^{5}-z^{4}-z^{3}-z-1$ \\
\hline & & $z^{83}-z^{91}$ \\
\hline \multirow{3}{*}{28} & \multirow{3}{*}{85} & $z^{28}-z^{26}-2 z^{22}-z^{21}-2 z^{10}-2 z^{9}-2 z^{6}-3 z^{5}+z^{4}-z^{2}-2 z+1$ \\
\hline & & $z^{26}+2 z^{23}+3 z^{22}+2 z^{21}+2 z^{11}+4 z^{10}+4 z^{9}+2 z^{8}+2 z^{7}+5 z^{6}+2 z^{5}+3 z^{2}+2 z$ \\
\hline & & $z^{85}-z^{88}$ \\
\hline \multicolumn{2}{|c|}{$(26,27,28)$} & $-z^{80}-z^{79}$ \\
\hline
\end{tabular}

